\title{
A Deep Learning-Based Intrusion Detection System for MQTT Enabled IoT
}

\author{
Muhammad Almas Khan ${ }^{1}$ (D), Muazzam A. Khan 1,*D, Sana Ullah Jan ${ }^{2} \mathbb{D}$, Jawad Ahmad 2,*D, \\ Sajjad Shaukat Jamal ${ }^{3} \mathbb{D}$, Awais Aziz Shah ${ }^{4}\left(\mathbb{D}\right.$, Nikolaos Pitropakis $^{2} \mathbb{D}$ and William J. Buchanan ${ }^{2}(\mathbb{D}$ \\ 1 Department of Computer Sciences, Quaid-i-Azam University, Islamabad 44000, Pakistan; \\ mkhan@cs.qau.edu.pk \\ 2 School of Computing, Edinburgh Napier University, Edinburgh EH10 5DT, UK; S.Jan@napier.ac.uk (S.U.J.); \\ N.Pitropakis@napier.ac.uk (N.P.); B.Buchanan@napier.ac.uk (W.J.B.) \\ 3 Department of Mathematics, College of Science, King Khalid University, Abha 61413, Saudi Arabia; \\ shussain@kku.edu.sa \\ 4 Department of Electrical and Informational Engineering (DEI), Polytechnic University of Bari, \\ 70125 Bari, Italy; awais.shah@poliba.it \\ * Correspondence: muazzam.khattak@qau.edu.pk (M.A.K.); J.Ahmad@napier.ac.uk (J.K.)
}

Citation: Khan, M.A.; Khan, M.A.; Jan, S.U.; Ahmad, J.; Jamal, S.S.; Shah, A.A.; Pitropakis, N.; Buchanan, W.J. A Deep Learning-Based Intrusion Detection System for MQTT Enabled IoT. Sensors 2021, 21, 7016. https:// doi.org/10.3390/s21217016

Academic Editors: Nancy Alonistioti, Spyros Panagiotakis and Evangelos K. Markakis

Received: 20 September 2021

Accepted: 19 October 2021

Published: 22 October 2021

Publisher's Note: MDPI stays neutral with regard to jurisdictional claims in published maps and institutional affiliations.

Copyright: (c) 2021 by the authors. Licensee MDPI, Basel, Switzerland. This article is an open access article distributed under the terms and conditions of the Creative Commons Attribution (CC BY) license (https:/ / creativecommons.org/licenses/by/ $4.0 /)$.

\begin{abstract}
A large number of smart devices in Internet of Things (IoT) environments communicate via different messaging protocols. Message Queuing Telemetry Transport (MQTT) is a widely used publish-subscribe-based protocol for the communication of sensor or event data. The publishsubscribe strategy makes it more attractive for intruders and thus increases the number of possible attacks over MQTT. In this paper, we proposed a Deep Neural Network (DNN) for intrusion detection in the MQTT-based protocol and also compared its performance with other traditional machine learning (ML) algorithms, such as a Naive Bayes (NB), Random Forest (RF), k-Nearest Neighbour (kNN), Decision Tree (DT), Long Short-Term Memory (LSTM), and Gated Recurrent Units (GRUs). The performance is proved using two different publicly available datasets, including (1) MQTT-IoTIDS2020 and (2) a dataset with three different types of attacks, such as Man in the Middle (MitM), Intrusion in the network, and Denial of Services (DoS). The MQTT-IoT-IDS2020 contains three abstract-level features, including Uni-Flow, Bi-Flow, and Packet-Flow. The results for the first dataset and binary classification show that the DNN-based model achieved 99.92\%, 99.75\%, and 94.94\% accuracies for Uni-flow, Bi-flow, and Packet-flow, respectively. However, in the case of multi-label classification, these accuracies reduced to $97.08 \%, 98.12 \%$, and $90.79 \%$, respectively. On the other hand, the proposed DNN model attains the highest accuracy of $97.13 \%$ against LSTM and GRUs for the second dataset.
\end{abstract}

Keywords: MQTT; IDS; IoT; security; classification

\section{Introduction}

Internet-of-Things (IoT) augments the physical objects (usually referred to as IoT nodes) with internet connectivity such that they can collect and share data with other nodes in the network without human interventions. To enable the secure and reliable exchange of data among IoT nodes, different communication and messaging protocols have been developed, such as Constrained Application Protocol (CoAP), Advanced Message Queuing Protocol (AMQP), Message Queuing Telemetry Transport (MQTT), and Extensible Messaging Presence Protocol (XMPP) [1]. Among all, MQTT has been widely used in smart homes [2-4], agricultural IoT [5,6], and industrial applications [7], etc. The reasons include support for communication on low bandwidths, low memory requirements, and reduced packet loss $[1,8,9]$.

MQTT communication protocol consists of four major components, including broker (central device), clients (IoT nodes), topic, and message. The topic in the MQTT protocol 
also contains information about the source and destination nodes of transmission messages among the networks. These topics are structured using the forward slash delimiter $(/)$ with messages consisting of data gathered by IoT sensors. Using the MQTT messaging transport protocol, every node has three main associated tasks: topic selection, topic publication, and topic subscription $[9,10]$. Primarily, the IoT nodes (clients) of MQTT communicate with each other via a central node called a broker; a broker can be working on the edge, i.e., a local broker, or it can be on the cloud, i.e., a remote broker. It allows IoT nodes to publish or subscribe topics or publish and subscribe at the same time if the node functionality allows, as shown in Figure 1 in general. For example, a Passive Infrared Sensor (PIS) for motion detection publishes sensed data to the broker that is subscribed by a camera. As soon as the PIS detects motion, the information is sent to the camera for further action.

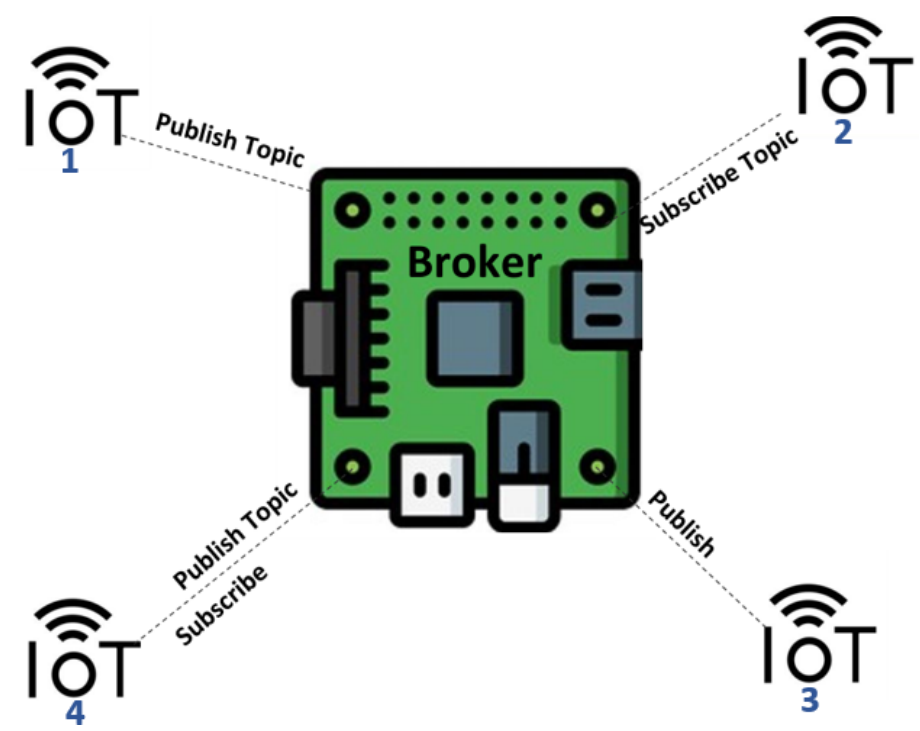

Figure 1. IoT communication via MQTT protocol.

The IoT has been identified as the most vulnerable network to be attacked by external, as well as internal, attackers [11-13]. External attackers try to corrupt the system from outside the networks. On the contrary, internal attackers operate from inside the network under threat. The internal attackers, however, can access information easily as compared to external attackers. In either case, prior to initiating an attack, the attackers usually gather information to check the vulnerability of the network or system using different tools, such as Masscan, Network Mapper (NMAP), or Shodan [14]. For example, the paper [15] presents a case scenario of an attacker using penetrating testing tools to collect information of brokers through the Shodan tool. Furthermore, the Shodan tool provided connection codes that indicate whether a broker needs authentication or not.

The different types of threats to the broker in an MQTT protocol are illustrated in Figure 2. As an example, by breaching the broker security and making to all topics, an attacker can expose critical information of the system. Similarly, if an attacker publishes a topic the same as any other publisher, it can control the subscribers of a given topic [15]. For example, streetlights can be subscribers to a valid publisher in a smart streetlight system [16]. An attacker connected to its broker can generate and send the wrong information over to control these streetlights. In addition, an internal attacker can compromise the integrity of MQTT data packets as they can have an opportunity of analysing and modifying them.

Studies show that attackers usually target central communication devices, i.e., brokers, in MQTT-based IoT systems. Denial-of-Service (DoS) [17], Man-in-the-Middle (MitM), scanning, and Intrusion are a few examples of common attacks on brokers [15,17-19]. In principle, the MQTT client starts a connection with a broker by sending a connect packet, and, since MQTT works on top of TCP/IP, the broker sends connection acknowledgement 
(connack). After receiving acknowledgement, the client starts data transmission to the broker. MQTT protocol can provide three levels of Quality of Service (QoS) that define the level of agreement and the assurance of successful communication between a transmitter and receiver in the network. The QoS level 0 has no acknowledgement mechanism in communication between the sender and receiver. [20]. In addition, an internal attacker sends multiple messages with QoS1 and QoS2 to make the broker busy in acknowledgements, thereby imposing a DoS attack [18].

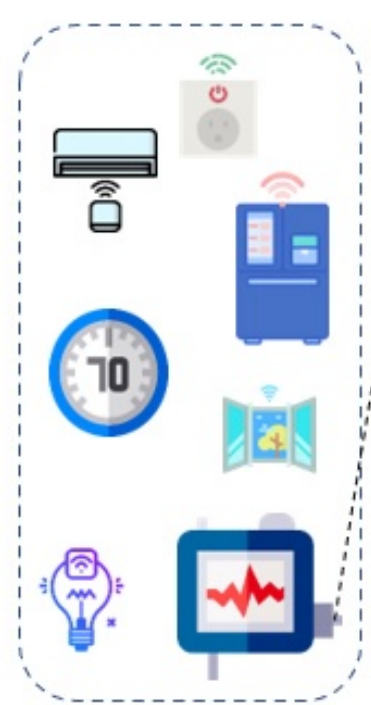

Subscribers

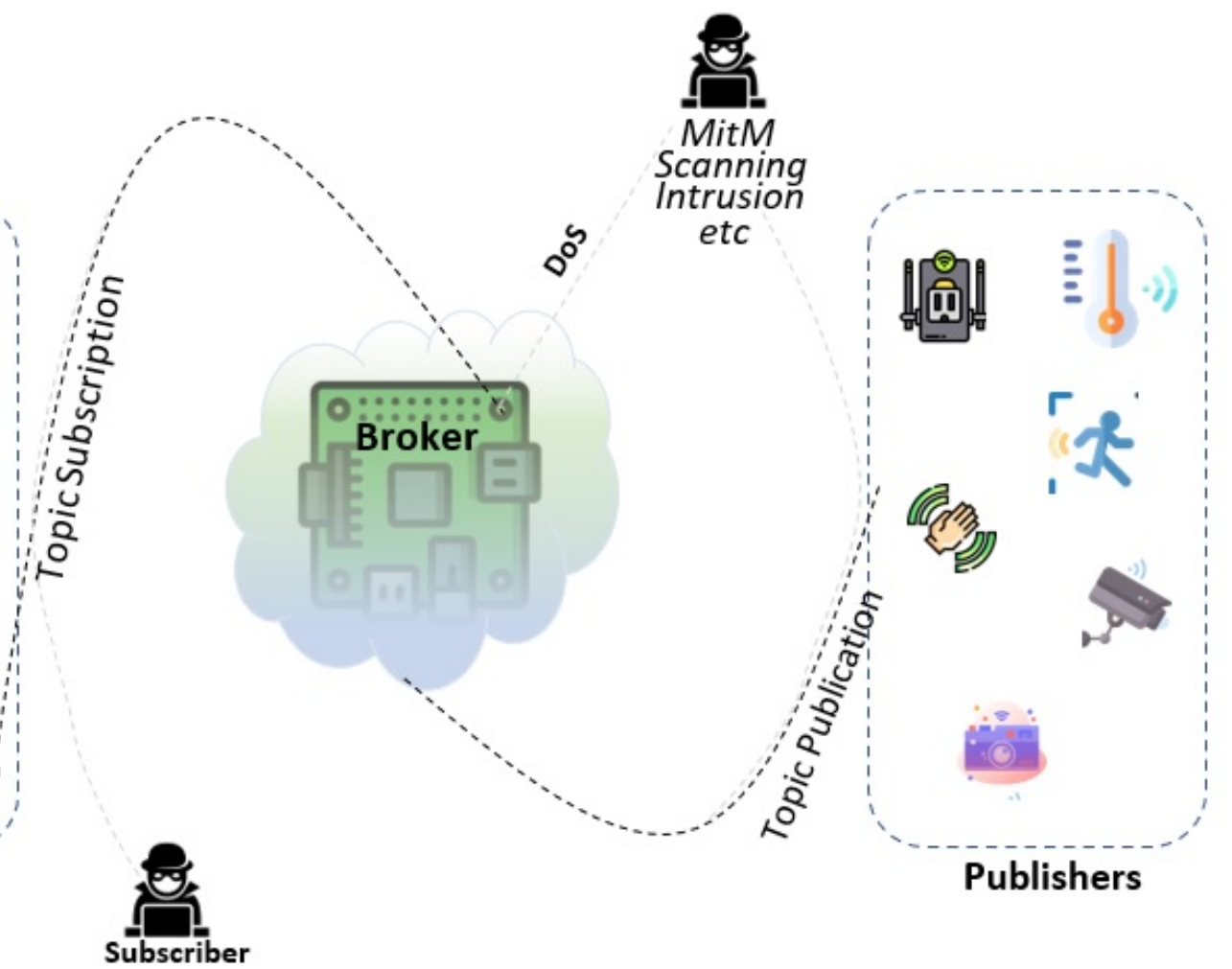

Figure 2. Attack scenario over MQTT protocol.

Machine learning (ML) has shown efficiency in different application areas, including intrusion detection systems for IoT [21-23]. Some researchers opine that ML has the potential to not only efficiently detect but also predict the attacks given efficient data have been used to train them. Therefore, in this paper, we propose an Intrusion Detection System (IDS) for MQTT protocol based on the ML algorithm, i.e., a Deep Neural Network (DNN). The proposed DNN algorithm is evaluated on the latest dataset named MQTT-IoT-IDS2020 and the dataset (https: / /joseaveleira.es/dataset; access date was 8 July 2021) discussed in [24] that contains three well-known attacks: MitM, Intrusion, and DoS over MQTT. The selected datasets [24,25] are generated in an MQTT simulated environment. In MQTT-IoTIDS2020, there are three abstract-level features, such as Packet-flow, Uni-flow, and Bi-flow, as mentioned in [25]. Detailed statistics of this dataset will be found in the upcoming section of this paper. The contributions can be summarised as follows.

- $\quad$ A DNN is proposed in this work for intrusion detection in MQTT-based protocol. Additionally, a number of ML models have been evaluated and compared for three different scenarios, including Bi-flow, Uni-flow, and Packet-flow, of abstract levels in the MQTT-IoT-IDS2020 dataset. The evaluation has been performed for binary as well as multi-class classification.

- The performance of the proposed DNN model is also evaluated for different attacks, including DoS, Intrusion, and MitM, in another dataset [24]. 
The remainder of this paper is organised as follows. Section 2 presents a literature review and a detailed discussion about related works. Section 3 provides a detailed explanation of the proposed intrusion detection system and other classical ML models. Section 4 illustrates the experimental setup, dataset selection criteria, results, and a discussion of the results. Section 5 concludes the paper and highlights potential future directions.

\section{Related Works}

IoT security is an open research area currently being addressed by researchers around the globe. Different security-enhancing methods have been proposed to protect IoT against anomalous adversarial attacks. These methods commonly aim at detecting intruders in the network by monitoring network activities, such as data flow rate. Here, a short literature review is presented to put forward current advances in IoT security, with a focus on intrusion detection systems targeting the MQTT messaging protocol of IoT. The authors of [26] presented an attack detection strategy for MQTT protocols based on a process tree. It models the network behaviour in terms of hierarchical branches of a tree, where it is further applied to detect attacks or anomalous behaviours. The model is evaluated using a detection rate where a total of four common types of attacks are induced in the network. However, newly developed adversarial attacks and intrusions have not been addressed. Furthermore, the paper [27] presents a fuzzy logic-based intrusion detection model specifically designed for protecting IoT nodes with the MQTT protocol against DoS attacks. Although fuzzy logic has shown its efficiency for different applications, including sensor fault detection in IoT [28]; however, its high complexity with an increase in the input dimension limits its potential in intrusion detection for IoT where huge data are transferred continuously. In addition, more advanced and complex attacks have been left untouched in paper [27] that raises questions over the efficiency of the proposed model for detecting other types of attacks.

ML and DL has shown efficiency in detecting complex and unknown intrusions, such as MitM, DoS, etc. [23]. Commonly used algorithms include Support Vector Machine (SVM) [29,30], Semi-supervised Spatio-Temporal Deep Learning Intrusions Detection (SSDeep-ID) [31], and Deep Feed-Forward Neural Network (DFFNN), ref. [32] etc.

In [33], multiple ML algorithms, including Autoencoder, RF, K-Means clustering, and Isolation Forest (IF), are employed to detect attacks in the IoT. However, the paper does not present clarity about the type of attacks considered in this work. In addition, the authors developed and evaluated an intrusion detection strategy on the network layer of the IoT that is not necessarily based on the MQTT messaging protocol.

Faker and Dodge [32] proposed a DL-based network intrusion detection system and evaluated it against CIC-IDS2017 and UNSW-NB15 datasets where accuracy and prediction time are used as evaluation metrics. The results show the significance of applying deep learning (DL) algorithms while designing intrusion detection systems for IoT. A total of 32 attack types from CIC-IDS2017 and UNSW-NB15 were included in the experiment using accuracy and prediction time as evaluation metrics. CIC-IDS2017 and UNSW-NB15 datasets are general purpose datasets not representing MQTT specifically. In [34], authors have worked on a new dataset known as MQTTset and proposed various ML algorithms for intrusion detection. 
In [35], performances of eight different ML algorithms, including DNN, Logistic Regression (LR), NB, SVM, Adaptive Boosting (AB), kNN, DT, and RF, are analysed against six datasets, such as KDD-99, NSL-KDD, UNSW-NB15, Kyoto2006+, and WSN-DS CICIDS2017. Intuitively, the DL achieved the best accuracy as compared to classical ML classifiers at the cost of the high computational requirements. This paper also does not address MQTT messaging protocol-related issues.

Tables 1 and 2 summarise intrusion detection systems proposed in recent literature with a tick $(\mathcal{V})$ in the last column indicating the given model is developed targeting the MQTT protocol. The first four columns show the reference number for the paper, the ML model exploited the evaluation method, and the evaluation metrics, respectively.

Table 1. Intrusion detection systems for MQTT protocol.

\begin{tabular}{|c|c|c|c|c|}
\hline Paper Ref & Method Used & Evaluation Method & Evaluation Metrics & MQTT Protocol \\
\hline [26] & Process Tree & Network modeling & Detection rate & 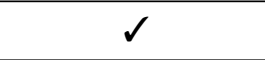 \\
\hline [27] & Fuzzy Logic & Network traffic & $\begin{array}{l}\text { Detection efficiency } \\
\text { Detection rate } \\
\text { Detection accuracy } \\
\text { False positive ratio }\end{array}$ & $\checkmark$ \\
\hline [31] & Deep Learning(DL) & $\begin{array}{l}\text { CIC-IDS2017 } \\
\text { CIC-IDS2018 }\end{array}$ & $\begin{array}{l}\text { Accuracy, Precision } \\
\text { Recall, F1 score }\end{array}$ & $x$ \\
\hline [25] & $\begin{array}{c}\text { Gaussian NB } \\
\text { LR } \\
\text { KNN } \\
\text { SVM } \\
\text { DT } \\
\text { RF }\end{array}$ & MQTT-IoT-IDS2020 & $\begin{array}{l}\text { Accuracy } \\
\text { Precision } \\
\text { Recall } \\
\text { F1-score }\end{array}$ & $\checkmark$ \\
\hline [34] & $\begin{array}{c}\text { RF } \\
\text { NB, Neural network } \\
\text { DT } \\
\text { Gradient boost } \\
\text { Multi-Layer Perceptron (MLP) }\end{array}$ & MQTTset & $\begin{array}{c}\text { Accuracy } \\
\text { F1-score } \\
\text { Confusion matrix }\end{array}$ & $\checkmark$ \\
\hline [35] & $\begin{array}{c}\text { NB, SVM } \\
\text { Adaptive Boosting } \\
\text { KNN } \\
\text { DT } \\
\text { RF }\end{array}$ & $\begin{array}{l}\text { UNSW-NB15 } \\
\text { Kyoto2006+ } \\
\text { WSN-DS } \\
\text { CICIDS2017 }\end{array}$ & $\begin{array}{l}\text { Accuracy } \\
\text { Precision } \\
\text { Recall } \\
\text { F1-score } \\
\text { RoC,AUC }\end{array}$ & $\mathrm{x}$ \\
\hline [36] & $\begin{array}{c}\text { NB, SVM } \\
\text { DT } \\
\text { SVM-RBM, GRU } \\
\text { Text-CNN }\end{array}$ & $\begin{array}{l}\text { ADFA-LD } \\
\text { KDD-99 }\end{array}$ & $\begin{array}{l}\text { Precision } \\
\text { Recall } \\
\text { F1-score }\end{array}$ & $x$ \\
\hline [37] & $\begin{array}{c}\text { CNN-LSTM, BLSTM } \\
\text { Ensemble, DNN } \\
\text { MLP } \\
\text { DT }\end{array}$ & $\begin{array}{l}\text { CIDDS-001 } \\
\text { UNSWNB15 } \\
\text { KDD-99 }\end{array}$ & $\begin{array}{l}\text { Accuracy } \\
\text { Specificity } \\
\text { Sensitivity }\end{array}$ & $x$ \\
\hline
\end{tabular}


Table 2. Review of Intrusion detection systems for MQTT protocol.

\begin{tabular}{|c|c|c|c|c|}
\hline Paper Ref & Method Used & Evaluation Method & Evaluation Metrics & MQTT Protocol \\
\hline [24] & $\begin{array}{c}\text { XGBoost } \\
\text { GRUs } \\
\text { Neural network } \\
\text { LSTM }\end{array}$ & Intrusion dataset & $\begin{array}{c}\text { Log loss } \\
\text { Cross entropy } \\
\text { Accuracy } \\
\text { F-beta score }\end{array}$ & $\checkmark$ \\
\hline [38] & $\begin{array}{c}31 \mathrm{ML} \text { algorithms } \\
\text { supervised and } \\
\text { Unsupervised }\end{array}$ & CICIDS2017 & $\begin{array}{c}\text { Accuracy } \\
\text { precision and recall } \\
\text { F-beta score } \\
\text { True positive } \\
\text { negative rate }\end{array}$ & $x$ \\
\hline [32] & $\begin{array}{l}\text { Deep Learning } \\
\text { Gradient boosting } \\
\text { Random Forest }\end{array}$ & $\begin{array}{c}\text { CICIDS2017 } \\
\text { UNSW-NB15 }\end{array}$ & $\begin{array}{c}\text { Accuracy } \\
\text { prediction time }\end{array}$ & $x$ \\
\hline [39] & CNN-GRUs & $\begin{array}{c}\text { MQTT-IoT-IDS2020 } \\
\text { BoT-IoT }\end{array}$ & $\begin{array}{c}\text { Accuracy } \\
\text { precision and recall } \\
\text { F-score }\end{array}$ & $x$ \\
\hline Proposed & DNN & $\begin{array}{l}\text { MQTT-IoT-IDS2020 } \\
\text { Intrusion dataset }\end{array}$ & $\begin{array}{c}\text { Accuracy } \\
\text { Precision } \\
\text { Recall } \\
\text { F1-measure }\end{array}$ & $\checkmark$ \\
\hline
\end{tabular}

In [24], a number of ML algorithms, such as eXtreme Gradient Boosting (XGBoost), GRUs, and LSTM, are used to design security models for the MQTT protocol in IoT. For the verification of the proposed algorithms, the author's used the MQTT dataset containing three types of attacks such as intrusion (illegal entry), DoS, and MitM. Different ML algorithms, such as NB, RF, DT, LR, KNN and SVM, are evaluated using the MQTT-IoTIDS2020 dataset [25]. The acceptable performance of these ML models for the proposal of the MQTT intrusion detection system was reported. The author of [40] proposed a single-layer ANN-based model for intrusion detection in an MQTT-enabled IoT system. The proposed model is evaluated on the KDD-99 and NSL-KDD dataset with acceptable performance measures. However, these datasets do not represent the MQTT-enabled IoT system-based environment. In [39], the author proposed a model for anomaly-based IDS in IoT systems using a Convolutional Neural Network (CNN) and GRUs for MQTT-IoTIDS2020. This study presents a comparison of several ML-based models for intrusion detection in MQTT-enabled IoT systems with the proposed DNN.

\section{Proposed Deep Neural Network (DNN) Based Intrusion Detection System}

Deep learning (DL) is a sub-field of machine learning inspired by the biological brain. These algorithms, also known as Artificial Neural Networks (ANNs), have better predictive capabilities as compared to conventional Multi-Layer Perceptron (MLP) because of a higher number of hidden layers. Primarily, ANNs consist of neurons connected with a neighbouring layer, which processes the input data using activation functions [41] in order to predict the output. Our proposed model consists of an input layer, two fully connected hidden layers, and an output layer. The data processing from input through the hidden layer to the output layer follows forward and backward propagation. Figure 3 shows the framework of DNN-based IDS for attack classification. The output layer is different depending upon the classification task, such as binary or multi-class. The input layer of our proposed DNN-based learning model takes into account the features of the MQTT protocol-based network, two hidden layers with Rectified Linear Unit (ReLU) activation, and an output layer with sigmoid activation in the case of binary classification and softmax for multi-class attacks classification. The reason behind choosing the softmax 
for multi-classification is based on our experimental results performed in this paper. As the MQTT-IoT-IDS2020 dataset contains three abstract-level features of MQTT protocol, i.e., Packet-flow, Bi-flow, and Uni-flow data. The proposed model is tested for all of the three mentioned features of MQTT contained in MQTT-IoT-IDS2020.

Figure 4 shows the number of input neurons, hidden layer, and output neurons. The data from the input layer is forward propagated through the hidden layer neurons during model training and backward propagated to update the weights and reduce the loss function until the model learns the proper weights and bias. Mathematically, the processing of data through the dense layer of neurons can be expressed as: $O: \mathbb{R}^{m} \times \mathbb{R}^{n}$, where $\mathrm{m}$ represents the input vector size, while $\mathrm{n}$ is the size of the output vector. Suppose $\mathrm{X}$ presents the input vector such that $\mathrm{X}=x_{1}, x_{2}, x_{3} \ldots x_{m-1}, x_{m}$, then the mathematical computation of the hidden layer can be expressed as a product of weights and an addition of bias as in the following equation:

$$
h_{i}(X)=f\left(W_{i}^{T} X+b_{i}\right)
$$

where $h_{i}$ is defined as $h_{i}: \mathbb{R}^{d_{i}-1} \rightarrow \mathbb{R}^{d_{i}}, \mathrm{f}$ is function from $f: \mathbb{R} \rightarrow \mathbb{R}$ defined by (2a) for the hidden layer. In Equation (1), the $b_{i} \in \mathbb{R}^{d_{i}}$ presents the bias that add to the product input and weights, i.e., $W_{i} \in \mathbb{R}^{d \times d_{i}-1}$.

$$
\begin{gathered}
\operatorname{ReLU}=\max (0, x) \\
\operatorname{Softmax}\left(x_{i}\right)=\frac{e^{x_{i}}}{\sum_{1}^{n} e^{x_{j}}} \\
\text { Sigmoid }=\frac{1}{1+e^{-x}}
\end{gathered}
$$

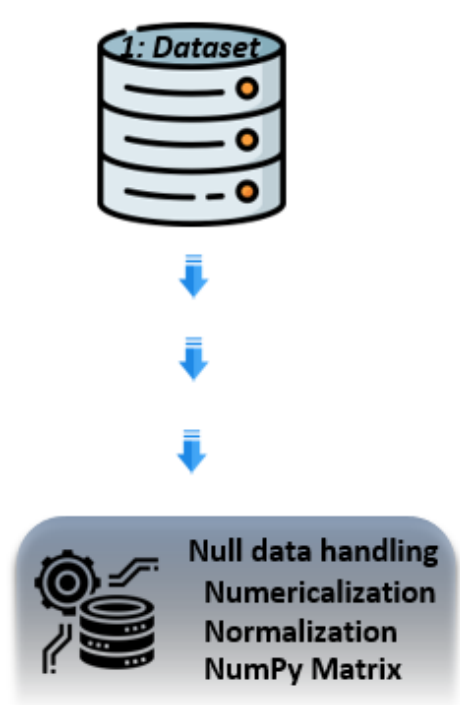

2: Data Pre-Processing

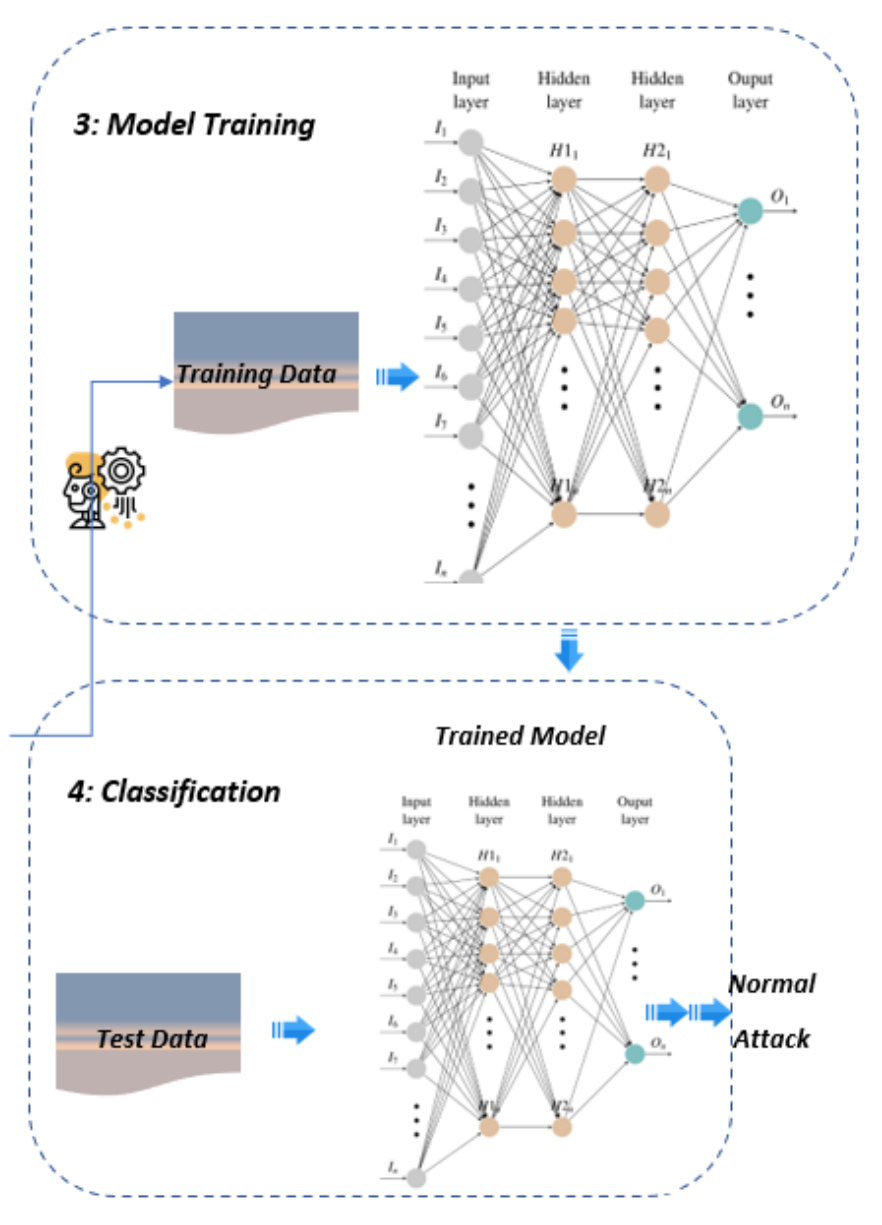

Figure 3. Proposed IDS framework. 
An Artificial Neural Network (ANN) consists of many stacked hidden layers that become a deep network. In general, these hidden layers can be expressed mathematically via Equation (3).

$$
H(X)=H_{l}\left(H_{l-1}\left(H_{l-2}\left(\ldots\left(H_{1}(X)\right)\right)\right)\right.
$$

Our proposed model is tested for binary as well as multi-class attack classification. Therefore, two different activation functions at the output layer are used. For binary classification, $\hat{y}$ is calculated at the last layer via sigmoid, as presented with mathematical expression in Equation (2c). Depending on the classification task, we utilised different cost functions; for binary attack classification, we used binary cross-entropy, as presented in Equation (4), while in the case of multi-attack classification, we utilised categorical cross-entropy, as presented in Equation (5). The loss function calculated the amount of difference between predicted labels and actual labels. The smaller the reduction in the loss function, the more accurate the prediction of the model. Optimisation algorithms play the main role in finding parameters in order to minimise or maximise any mathematical functions. In deep learning, such optimisation algorithms helps to reduce the cost function for particular. Out of many existing optimisation algorithms used in deep learning, we adopted Adaptive Moment Estimation (ADAM) as an optimiser to reduce the cost function of our proposed model. The ADAM optimiser combines the best feature Root Mean Square Propagation (RMSProp) optimiser and momentum. That is why it is still the best optimiser in most DL-related tasks and is used in lots of optimisation problems in deep learning function:

$$
\begin{gathered}
J(y, \hat{y})=\frac{1}{t} \sum_{i=1}^{t}-\left(y_{i} \times \log (\hat{y})+(1-y) \times \log (1-\hat{y})\right) \\
J(y, \hat{y})=\frac{1}{t} \sum_{i}^{t} \sum_{j}^{c} y_{i j} \log (\hat{y})
\end{gathered}
$$

where $J$ is a function defined on $y$ and $\hat{y}, \hat{y}$ is predicted output calculated at the last layer by sigmoid or softmax of our proposed model, and $y$ is the actual label, $t$ is the batch size, and $c$ denotes the class category.

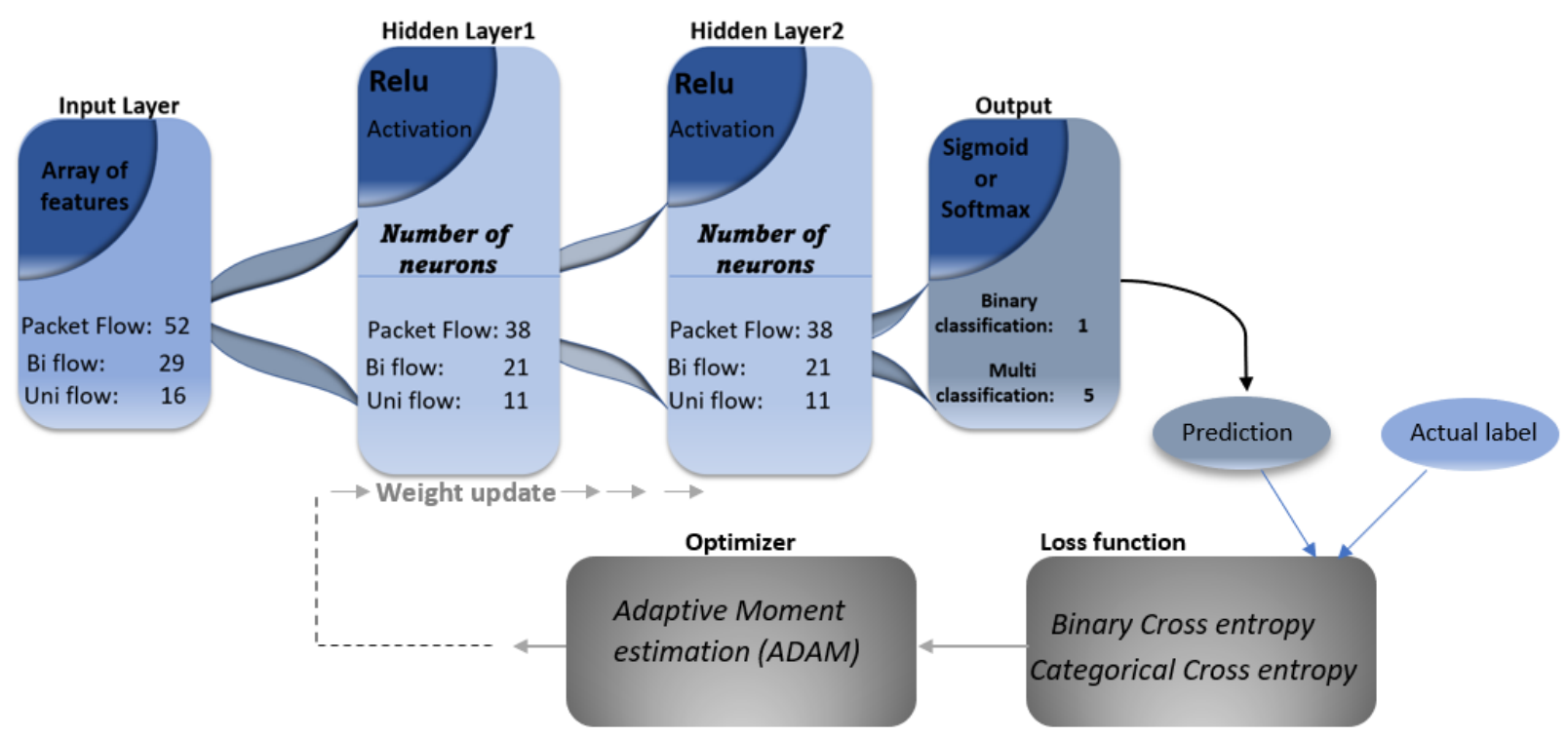

Figure 4. The proposed model's training setup for binary and multi-attack classifications.

\subsection{Other Classical ML Models}

This subsection highlights the brief theoretical concepts behind the other classical ML models that are used for cross-comparison in this study with the proposed Deep Neural Network model. 


\subsubsection{K-Nearest Neighbour}

This learning algorithm is categorised as a supervised learning model and known as a lazy learner because of the fact that it does not learn a discriminative function from the training data rather memorise it. For example, the weights during the training process of the logistic regression are learned. The KNN algorithm is relatively straightforward, the working of KNN can be summarised in the following three main points:

- Choose the number of $k$ neighbour and distance metrics.

- $\quad$ Locate the $k$ neighbour of the test sample.

- Assign label accordingly to the majority of the label in $k$ neighbour.

Different distance metrics exist such as Manhattan distance, Minkowski distance, and Euclidean distance, etc. Among all of these, the euclidean is widely used as a distance metric in KNN. The Euclidean distance and Manhattan distance is a specialised form of Minkowski. The mathematical representation of these distances is given as below in Equation (6).

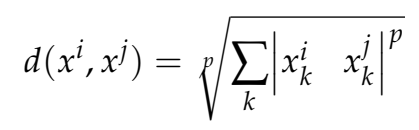

where the parameter $p$, if changes then the above equation change to other distance metrics. For example, if $p=2$ then the above equation becomes euclidean, and if $p=1$ then it becomes Manhattan distance.

\subsubsection{Decision Tree}

This model breaks our data into a hierarchical manner, so that to make predictions on new data, that is why due to this hierarchical learning style of this model it is called a decision tree. This learning model also belongs to supervised learning and can handle both classification \& regression problem. This model makes tree, where each node of the decision tree model represents an attribute and each leaf node represents a class label. The main working of the decision tree can be described as:

- Find the best attribute and place it in the root of the tree.

- Make subset of training data in such a way that each subset contains data with the same value for an attribute.

- Repeat above two steps until reach to the leaf node.

Assume a dataset consist of $\mathrm{n}$ attributes, for the selection of best attribute as the root node of the tree, researcher work on mathematical measures, these mathematical measured values are used for such attribute selection, these measures are information gain and Giniindex. Mostly the information gain is used when the attribute is categorical, while for continuous attributes the Gini index is favourable. An information gain is a reduction in entropy. Entropy is a measure used to calculates the randomness of data within attributes or features of a dataset. Mathematically entropy can be represented as bellow equation

$$
\text { Entropy }=\sum_{i=1}^{c}-p_{i} * \log _{2}\left(p_{i}\right)
$$

here $p_{i}$ denotes the proportion of the sample that belongs to class $c$. The following steps are used in calculating the information gain using entropy.

- Calculates the entropy of the target attribute.

- Calculates the entropy of other attributes and subtract from the entropy of target.

Mathematically it is represented as:

$$
\text { Information Gain }=E(\text { Target })-E(\text { Target, Attribute })
$$




\subsubsection{Random Forest}

Random Forest learning model is a type of supervised ML model. It is an ensemble model which makes use of multiple trees in predictions of a target. This model is used for regression and classification problems. It takes $\mathrm{n}$ samples as input and creates multiple trees based on a subset of input features. Then on the results of every tree, a majority voting is performed in order to get the final prediction for the target class variable.

Assume $\mathrm{m}$ denotes the total features in data, the main working of this learning model can be summarised in the following points.

- Select $k$ number of features randomly from $\mathrm{m}$ features of data such that $k<<\mathrm{m}$.

- Calculates the best split for $\mathrm{k}$ selected features.

- Split the node into child nodes using best split.

- Repeat above until leaf node reached.

- Build a forest of trees by repeating the above steps.

\subsubsection{Naive Bayes}

This learning model is based on Bayes rules in learning and predicting the new instances class label. Bayes theorem provides the way of calculating the posterior probability of class as depicted in Equation (9) below.

$$
p(c \mid x)=\frac{p(x \mid c) p(c)}{p(x)}
$$

where $p(c \mid x)$ indicates the posterior probability of the target class given independent variable $x, p(c)$ indicates the prior probability of the target class, $p(x \mid c)$ represents the likelihood and $p(x)$ is the prior probability of independent variable. In comparison to other, the NB performs better and fast prediction of the test set. This model performs better in multi-classification problems. sci kit-learn provides three types of models for Naive Bayes, these are Gaussian, Multinomial, Bernoulli.

\section{Experimental Setup and Results}

\subsection{Dataset Selection}

Dataset helps in evaluation of ML model performance against attack detection for the network.

Numerous datasets for the evaluation of IDS are proposed by researchers in the area of network security. That helps in understanding the performance of particular machine learning or deep learning algorithms for intrusion detection. We reviewed the existing publicly available dataset for IDS evaluation in order to find the best representative for our proposed IDS evaluation in MQTT enables IoT systems. There are two types of datasets available in network security. Table 3 compares the existing datasets available for evaluations and the representation of each dataset for MQTT protocol, whether a dataset is a general-purpose or special purpose. 
Table 3. Special and general purposed dataset for IDS evaluation.

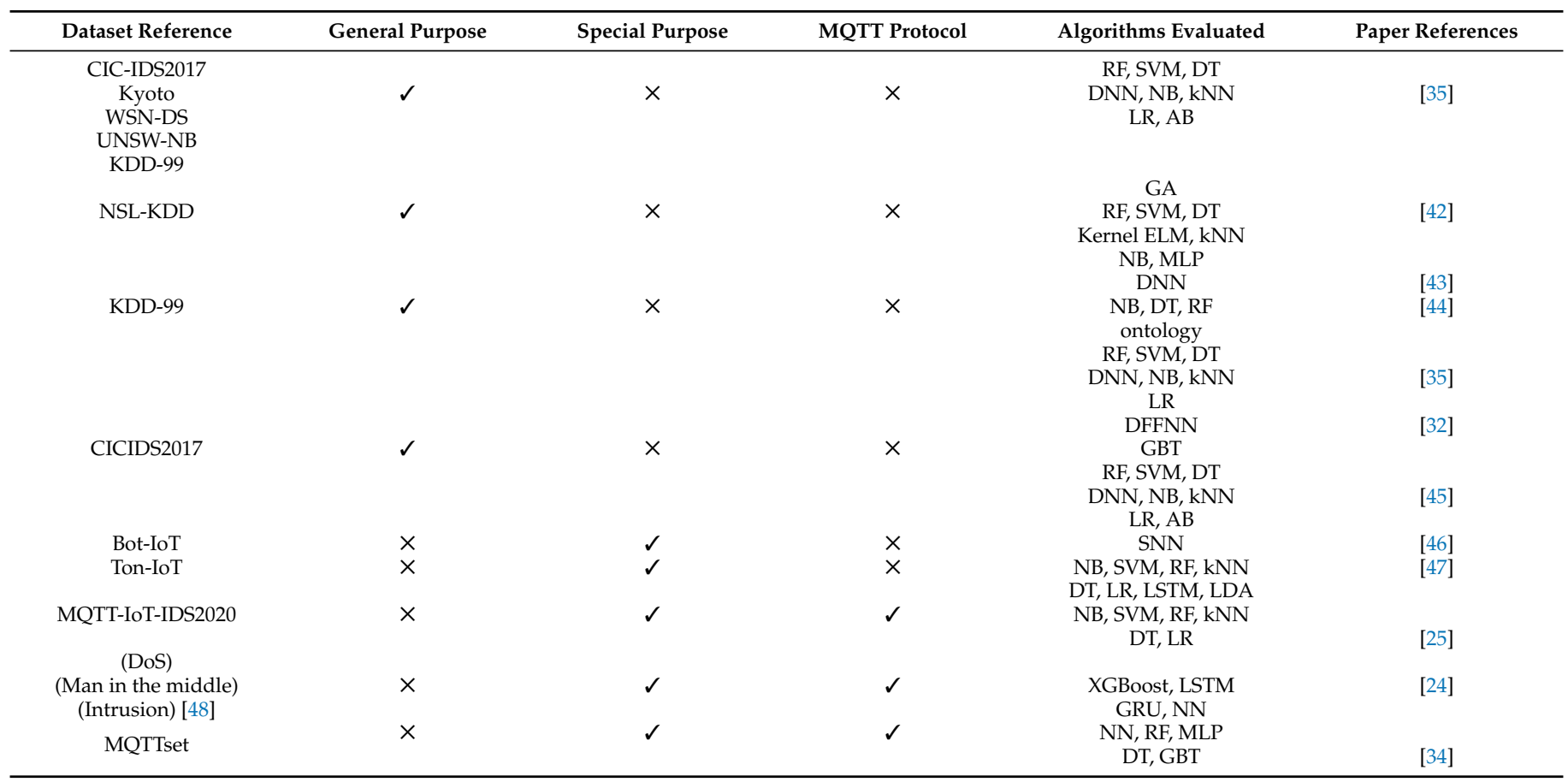

\subsubsection{General Purpose}

The general-purpose datasets are referred to as those which are generally used for IDS evaluation representing a general computer network. They are not created for specific networks nor replicating the specific types of IoT protocols. For example, Canadian Institute for Cybersecurity proposed several general-purpose NIDS datasets such as CICDoS dataset [49], CIC-IDS2017 [49] and CIC-IDS2018 [50], which helps the researchers community in intrusion detection to test the performance of ML or DL based learning models. In the literature, there are multiple datasets for general-purpose Network Intrusion Detection System (NIDS) evaluation, most common of them used for example KDD-99 [49], NSL-KDD [49] etc. The researcher evaluates the performance of the proposed IDS by using such a dataset for generalised networks. A lot of research exists over these datasets, that shows the significance of each ML, DL, or Ensemble Learning (EL).

\subsubsection{Special Purpose}

The dataset was created for special purposes to represent specific types of networks or protocols. There are some specialised networks, such as IoT-based [51], Internet of Vehicles (IoV) networks [52], and Supervisory Control and Data Acquisition (SCADA) network [53]. These networks are comprised of some specialised devices that can not be found in other networks. These devices are especially dedicated to such networks, for example, remote terminal units [54] in SCADA networks, etc. Table 2 shows the special and general-purpose datasets along with evaluated algorithms of very recent research. After our review, we selected the MQTT-IoT-IDS2020 dataset to check the performance of our proposed algorithm for the MQTT protocol. However, this table clearly shows that a Deep Neural Network for MQTT protocol is still an interesting idea. The focus of this work is to evaluate the Deep Neural Network for the MQTT protocol.

\subsubsection{Prepossessing and Description of Selected Datasets}

For our proposed IDS, we chose MQTT-IoT-IDS2020 [25] and the other latest published MQTT dataset (https: / / joseaveleira.es/ dataset; access date was 8 July 2021) [24] for testing the performance of the proposed DNN algorithm. There were sensors, a camera, and other devices that communicate with each other via the MQTT protocol while capturing these 
datasets. Tables 4 and 5 show the statistics of these two datasets used in the current study for the performance evaluation of the proposed IDS. These are the latest datasets and are created in an MQTT protocol-enabled simulated environment; hence, they better represent the features of the MQTT protocol-based IoT network.

Table 4. Three abstract-level feature statistics of MQTT-IoT-IDS2020.

\begin{tabular}{ccccc}
\hline & Classes & Total Instances & Normal & Attack \\
\hline \multirow{4}{*}{ Bi-flow } & Biflow_mqtt_bruteforce & 16,696 & 2152 & 14,544 \\
& Biflow_normal & 86,008 & 86,008 & $\times$ \\
& Biflow_scan_A & 25,693 & 5786 & 19,907 \\
& Biflow_scan_sU & 39,664 & 17,230 & 22,434 \\
& Biflow_sparta & 91,318 & 77,202 & 14,116 \\
\hline \multirow{5}{*}{ Uni-flow } & Uniflow_mqtt_bruteforce & 33,079 & 4205 & 28,874 \\
& Uniflow_normal & 171,836 & 171,836 & $\times$ \\
& Uniflow_scan_A & 51,358 & 11,561 & 39,797 \\
& Uniflow_scan_sU & 25,845 & 34,409 & 22,436 \\
& Uniflow_sparta & 182,407 & 154,175 & 28,232 \\
\hline Packet-flow & mqtt_bruteforce & $90,876,584$ & $70,980,732$ & $19,895,852$ \\
& Normal & $1,056,230$ & $1,056,230$ & $\times$ \\
& scan_A & 111,392 & 70,768 & 40,624 \\
& Biflow_scan_sU & 233,255 & 210,819 & 22,436 \\
& Biflow_sparta & $130,876,584$ & $90,980,732$ & $39,895,852$ \\
\hline
\end{tabular}

Table 5. MQTT dataset ${ }^{1}$ statistics.

\begin{tabular}{llll}
\hline File Name & Total & Normal & Attacked \\
\hline DoS.CSV & 94,625 & 49,112 & 45,513 \\
Intrusion.CSV & 80,893 & 78,995 & 1898 \\
MitM.CSV & 110,668 & 106,813 & 3855 \\
\hline
\end{tabular}

${ }^{1}$ https://joseaveleira.es/dataset.

Five scenarios were launched during the creation of the MQTT-IoT-IDS2020 dataset. These scenarios are normal operation, aggressive scan, UDP scan, Sparta SSH brute-force, and MQTT brute-force attack [25]. Each of these scenarios is recorded in separate files for three abstraction level network flow features of MQTT enabled simulated network. These flow features include Packet-flow, Uni-flow, and Bi-flow features. Every flow-level features of MQTT have five files representing attack and normal of particular scenario as mentioned above. For all of these Packet-flow, Bi-flow, Uni-flow data, we implemented a python script to combine all of these five files in each network flow-level into one combined CSV and create a combined dataset for each network flow-level feature. The combined CSV contains the binary label and multi-class label attribute in order to test the proposed algorithm performance over the MQTT protocol recorded traffic for binary as well as multi-attack classification. Figure 5 presents files of MQTT-IoT-IDS2020 in each network flow feature and their combined version dataset for each flow-level features of MQTT-IoT-IDS2020. For example, in Uni-flow feature data of MQTT, there are five files, and we combined all of these five files into one CSV with two extra columns with attack and the type of attack. The statistic of the combined version data for Packet-flow, Bi-flow, and Uni-flow is given in Figures 6-8 in the form of nested pie plot. The most outer plot shows the normal and attacked instances, and the inner pie plot shows the statistics of each of the five scenarios separately presenting the distribution of multi-type attacks. The reason behind merging and creating binary as well as multi-class combined version datasets is to test the proposed IDS performance on binary and multi-class attack classification for each type of network features, i.e., Packet-flow, Bi-flow, and Uni-flow [25]. The final prepossessed dataset is split into two parts: $80 \%$ for training and $20 \%$ for testing the trained ML model performance. 


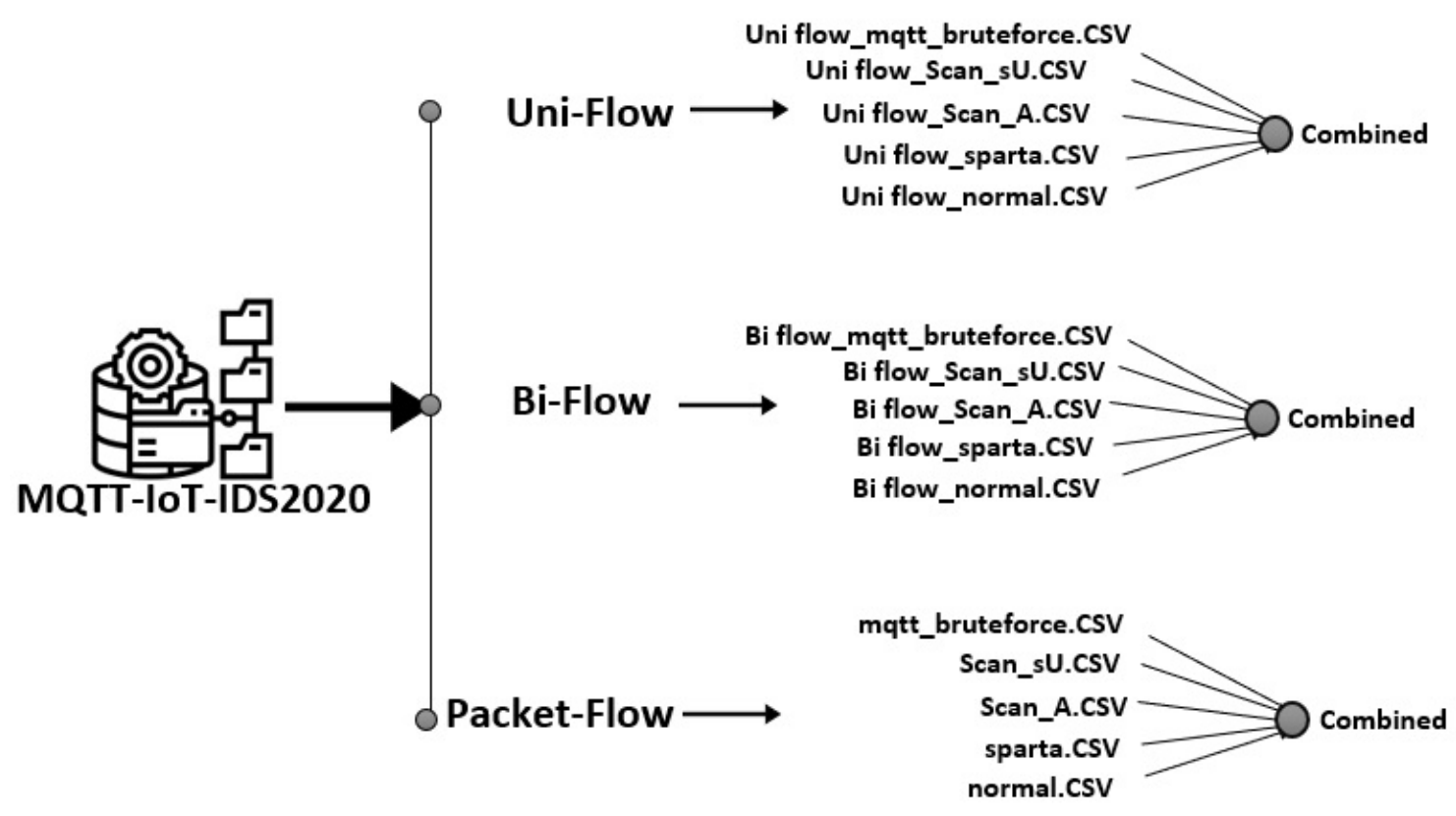

Figure 5. MQTT-IoT-IDS2020 pre-processing.

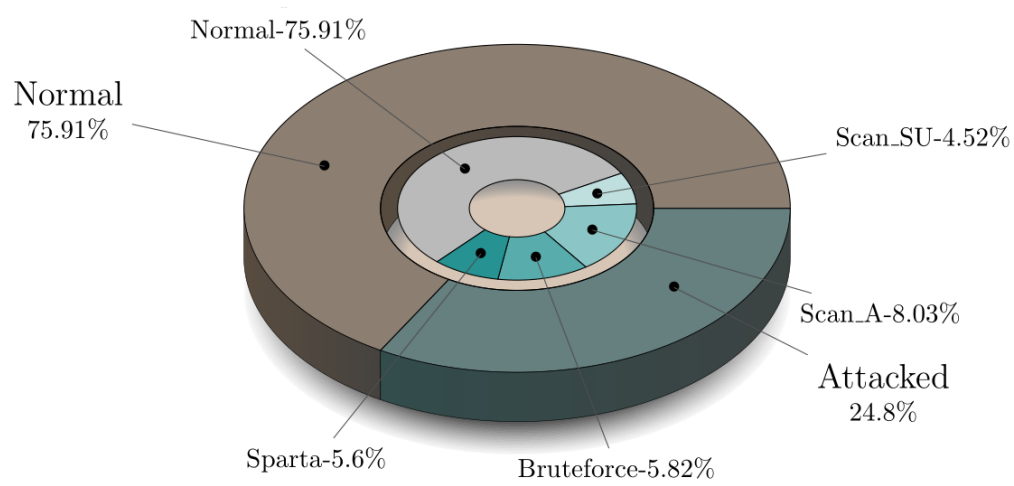

Figure 6. Five files statistics of Uni-flow.

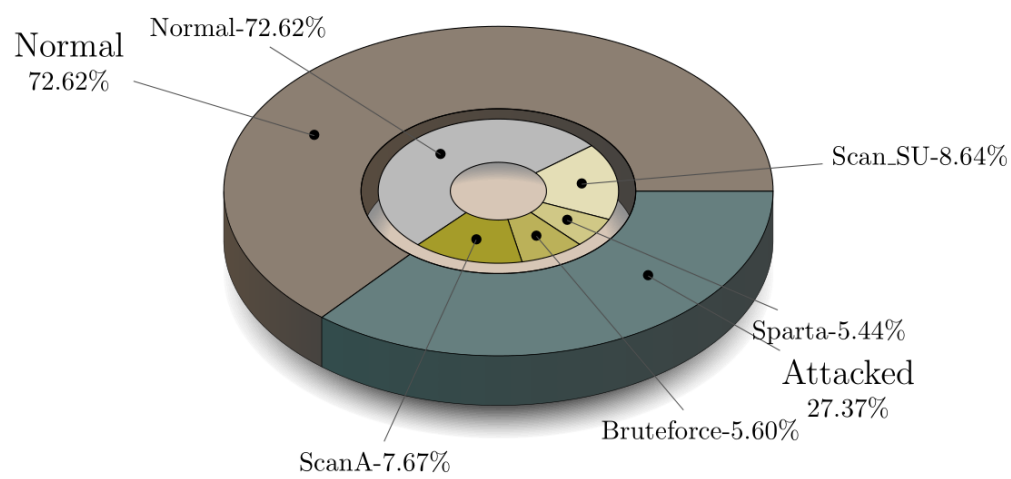

Figure 7. Five files statistics of Bi-flow. 


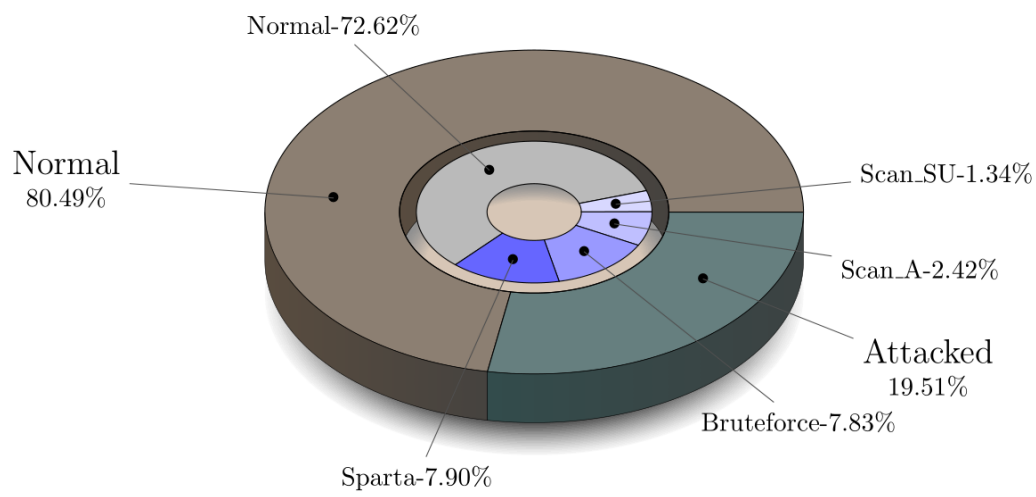

Figure 8. Five files statistics of Packet-flow.

In order to test the performance of the proposed DNN approach for IDS in an MQTTbased IoT system, we performed some pre-processing to the combined version of each feature level data in order to further prepare it. Because ML/ DL algorithms have requirements before applying these algorithms to data. All the features explanations of the dataset are available in detail in [25]. We analysed the data in each network flow-level features data of MQTT-IoT-IDS2020, i.e., Uni-flow, Bi-flow, and Packet-flow. As discussed above and mentioned in Figure 5, for each flow-level feature, all the attacked and normal scenarios were combined into one combined.csv file. Among all these, the Packet-flow features have some attributes that contained nulls value above 90 percent; these attributes are mqtt_flag_uname, mqtt_flag_passwd, mqtt_flag_retain, mqtt_flag_qos, mqtt_flag_willflag, mqtt_flag_clean, and mqtt_flag_reserved. We removed such attributes by threshold. For the remaining features in packet featured data, we put the median to fill the null-valued variable as the median is less susceptible to an outlier. Apart from this, we perform label encoding and one-hot label encoding as all TCP and IP flags were label encoded, and we perform one-hot encoding to express protocol features more clearly to our proposed model, due to which the packet feature increased as there were different protocols in the dataset. In Uni-flow and Bi-flow featured data, we removed certain features, such as source, destination IP address, and time stamp, etc. Besides this, certain features, such as MQTT flags, were removed in [25]; however, we have not removed such categorical features, but we instead prepared it by numerically encoding by the label encoder. Some features contained multi-type data, for example, string and int64 in one feature, so we converted the string into int64 representation in order to prepare for deep learning model. Features such as tcp_flag_res, tcp_flag_ns, tcp_flag_ecn, and protocols are converted, as and we performed label encoding as they are categorical in nature. Apart from this, we performed feature scaling to some features in Packet, Uni-flow, and Bi-flow data. Features such as mean_pkt_len, num_byte, min_pkt_len, max_pkt_len in Uniflow; fwd_num_byte, fwd_num_byte, fwd_max_pkt_len in Bi-flow; and ip_len in Packet-flow have scaled using feature scaling in python.

\subsection{Experimental Setups}

\subsubsection{Evaluation Metrics}

Understanding the model performance requires the statistical ground truth values, which measure how the model performs in attack classification from normal data. Several 
evaluation metrics can be used to check the performance of an IDS. These metrics are shown from Equation (10a-d).

$$
\begin{array}{r}
\text { Accuracy }=\frac{T P+T N}{T P+T N+F P+F N} \\
\text { Precision }=\frac{T P}{T P+F P} \\
\text { Recall }=\frac{T P}{T P+F N} \\
F 1-\text { measure }=2 \times\left(\frac{\text { Precision } \times \text { Recall }}{\text { Precision }+ \text { Recall }}\right)
\end{array}
$$

where:

- $\quad T P$ is the correct classification of normal as normal flow.

- $\quad T N$ is the correct classification of attacked as attacked flow.

- $\quad F P$ is the incorrect classification of normal as attacked flow.

- $\quad F N$ is the incorrect classification of attacked as normal flow.

Accuracy is the ratio of the correctly classified connection records to the complete test dataset. Machine learning (ML) or deep learning (DL) algorithms are considered to be the best model if their accuracy is high. Precision refers to the ratio of the accurate detection of attacked instances to the number of all detected attacked instances. This is the second measure for the evaluation of the machine learning algorithm: if the model is categorised high, it is a good model. A recall is another measure used to evaluate the ML algorithm performance that gives the relation between true positive $(T P)$ predictions to true positive $(T P)$ and false negative ( $F P)$ predictions. In calculating the F1 measure, the precision and recall are both used as a harmonic mean.

\subsubsection{Programming Libraries and Parameters Setup}

Keras (the Python deep learning API) with a hardware specifications Core i7 processor (16 GB RAM) is used in this work. The experiment was implemented in python 3.9.5 programming language using an interactive Python-based IDE named Jupyter notebook inside Anaconda distribution used as a software tool for the implementation and evaluation of proposed experiments. We used the pandas-profiling 2.11.0, which is an open-source module of python that provides the facility for data analysis. There are various things that have an effect on the results of the DL algorithm, for example, batch size, learning rate, type of optimiser for reduction of the loss function. The optimiser in designing the deep learning algorithms plays the main role because it reduces the cost function with less effort and less resource usage, but it depends on the nature of the optimiser. Accuracy increases as the reduction in the loss function. For the reduction in the loss function in our proposed model, i.e., binary-cross entropy or categorical-cross entropy, we studied and reviewed the existing optimiser, and we select the ADAM [55] optimiser out of the existing available optimisers because it will optimise the categorical cross-entropy loss function in the case of multi-classification, while binary-cross entropy will be optimised via ADAM in the case of binary classification in the proposed model.

ADAM combines the advantages of two Stochastic Gradient Descent (SGD) extensions, i.e., Adaptive Gradient Algorithm (Adagrad) [56] and Root Mean Square Propagation (RMSProp) [57]. ADAM implements momentum that brings smoothing and fast searching during training [57], and with the help of RMSProp optimiser, they change the learning rate efficiently during training time [57], which converges very quickly towards the global minima. Due to both of the mentioned advantages, we choose the ADAM as an optimiser in our proposed model. Certain studies have revealed that the learning rate and batch size 
have high correlations between each other [58-60], which is if there is a change in learning rate or in batch size, then the accuracy is impacted by such changes. In [58], it is clearly showed that when the learning rate is small, with the increase in batch size, the accuracy slows down, and while increasing the batch size with a slightly large learning rate, the accuracy increases. Therefore, we run multiple experiments to find the optimal batch size of our final model for binary as well as multi-attack classifiers. We summarised all of the results of different batch sizes, as shown in Tables 6-8. They also show a comparison among batch size performance of the classification MQTT. Out of our experiment with different batch sizes and different activation functions in the last layer that has the same hidden layer activation (relu) and network architecture, the higher batch for all three abstraction level features is recommended.

We tested the 32, 64, 128, and different activation functions in the last layer for binary as well as multi-attack classification. As the batch size increases, the accuracy increases. Out of all the experiments, the batch size of 128 proved optimal and gave good results in comparison to other lower batches. Based on the results in Tables 6-8, we recommend a higher batch size for the MQTT-IoT-IDS2020 dataset. Another advantage with the higher batch size is the number of iterations in each epoch decreases, which saves much of the learning time, and the model runs efficiently and fast.

Table 6. Binary-class attack classification with the sigmoid activation function in the output layer.

\begin{tabular}{cccc}
\hline Network Feature & Batch Size & Training Accuracy & Testing Accuracy \\
\hline \multirow{2}{*}{ Packet-flow } & 32 & 93.74 & 92.93 \\
& 64 & 93.43 & 93.43 \\
& 128 & 94.99 & 94.57 \\
\hline \multirow{2}{*}{ Bi-flow } & 32 & 99.32 & 99.29 \\
& 64 & 99.59 & 99.66 \\
& 128 & 99.99 & 99.92 \\
\hline \multirow{2}{*}{ Uni-flow } & 32 & 99.73 & 99.74 \\
& 64 & 99.63 & 99.77 \\
& 128 & 99.94 & 99.98 \\
\hline
\end{tabular}

Table 7. Multi-class attacks classification with sigmoid activation function on output layer.

\begin{tabular}{cccc}
\hline Network Feature & Batch Size & Training Accuracy & Testing Accuracy \\
\hline \multirow{2}{*}{ Packet-flow } & 32 & 88.66 & 88.62 \\
& 64 & 88.71 & 88.78 \\
& 128 & 89.51 & 89.71 \\
\hline \multirow{2}{*}{ Bi-flow } & 32 & 94.32 & 93.89 \\
& 64 & 93.59 & 94.06 \\
& 128 & 93.99 & 93.92 \\
\hline \multirow{2}{*}{ Uni-flow } & 32 & 94.13 & 93.99 \\
& 64 & 93.83 & 93.77 \\
& 128 & 94.04 & 93.98 \\
\hline
\end{tabular}


Table 8. Multi-class attacks classification with softmax activation function on output layer.

\begin{tabular}{cccc}
\hline Network Feature & Batch Size & Training Accuracy & Testing Accuracy \\
\hline \multirow{3}{*}{ Packet-flow } & 32 & 90.02 & 90.17 \\
& 64 & 90.71 & 90.78 \\
& 128 & 91.71 & 91.96 \\
\hline \multirow{2}{*}{ Bi-flow } & 32 & 97.32 & 98.89 \\
& 64 & 97.59 & 97.99 \\
& 128 & 98.02 & 98.34 \\
\hline \multirow{2}{*}{ Uni-flow } & 32 & 96.99 & 97.39 \\
& 64 & 97.97 & 97.87 \\
& 128 & 97.04 & 97.91 \\
\hline
\end{tabular}

\subsection{Performance Analysis and Discussion}

This section discusses the results obtained. Based on the experiments of Tables 6-8, we selected 128 batch size with a sigmoid output function at the last layer for binary classification, softmax for multi-classification, and the ADAM optimiser, which will optimise our cost function for binary classification as well as multi-classification of an attack for our proposed deep learning model toward intrusion detection in MQTT protocol-based smart IoT systems. Choosing a higher batch size reduces the iteration in each epoch, which saves a lot of time during model training. Due to the stochastic nature of the proposed algorithms, the results may vary each time the model runs because the weights are randomly assigned during training the model each time. Every time the model runs, it learns differently because of its stochastic nature. Furthermore, we performed a five-fold cross-validation evaluation on the proposed model to find the average evaluation metrics, i.e., accuracy, precision, recall, etc., with standard deviation. Tables 9 and 10 show the five-fold crossvalidated evaluation accuracy of our proposed model against binary as well as multi-class attack classification, respectively. For binary classification, the proposed model performed well on both Bi-flow and Uni-flow features data of MQTT with 99.753 and 99.927 mean accuracy, respectively. While in the case of Packet-flow data of MQTT, it performed with a mean accuracy equal to 94.943 . Figure $9 \mathrm{a}-\mathrm{f}$ shows learning curves of the five-fold binary attack classification and multi-attack classification in terms of loss and accuracy for each abstraction level of the feature of MQTT, i.e., Uni-flow, Bi-flow, and Packet flow.

Table 9. Binary-class attack classification with five-fold cross validation results in terms of accuracy.

\begin{tabular}{cccc}
\hline Network Features & Accuracy & Mean & Standard Deviation \\
\hline \multirow{3}{*}{ Packet-flow } & 94.996 & & \\
& 94.907 & & 0.034 \\
& 94.936 & 94.943 & \\
94.966 & & \\
& 94.911 & & 0.029 \\
Bi-flow & 99.788 & & \\
& 99.730 & 99.753 & \\
& 99.711 & & \\
& 99.778 & & \\
& 99.759 & & \\
\hline & 99.9333 & & \\
& 99.929 & & \\
& 99.950 & & \\
& 99.869 & & \\
& 99.953 & & \\
\end{tabular}


Table 10. Multi-class attacks classification results with five-fold cross validation in terms of accuracy.

\begin{tabular}{|c|c|c|c|}
\hline Network Features & Accuracy & Mean & Standard Deviation \\
\hline Packet-flow & $\begin{array}{l}91.900 \\
91.022 \\
85.932 \\
92.524 \\
92.615\end{array}$ & 90.798 & 2.498 \\
\hline Bi-flow & $\begin{array}{l}98.063 \\
97.966 \\
98.340 \\
98.275 \\
97.993\end{array}$ & 98.127 & 0.154 \\
\hline Uni-flow & $\begin{array}{l}96.872 \\
96.916 \\
97.549 \\
96.306 \\
97.779\end{array}$ & 97.084 & 0.524 \\
\hline
\end{tabular}

The quick convergence of the model can be observed. Learning curves help in understanding and diagnosing an over-fit, under-fit, or well-fit model on the training and testing datasets. A model is said to be under-fitted in two cases identified from the learning curve: in case 1, the training loss curve will form a straight line, i.e., no change, and in case 2, the model loss will continuously decrease at the end of the model training process. While in over-fitting, the testing loss begins to increase after some time faster than the training loss curve. The well-fit model shows a different learning curve than over-fitted and under-fitted models. That is, a model is said to be well-fit if the training and testing curves have a small gap or it decreases to a point of stability. Similarly, as mentioned above, we can understand the model performance on the MQTT-IoT-IDS2020 dataset by observing Figure 9a-f. Packet-flow accuracy is less than the others, i.e., Uni-flow and Bi-flow. One of the reasons for low accuracy on Packet-flow feature data of MQTT is the imbalance of the attack classes' distribution of Packet-flow data in each file. In order to reduce the imbalanced class problem, we have selected the data from five files of Packet-flow feature data of the MQTT-IoT-IDS2020 dataset. We further evaluate our model in order to test its performance on other evaluation metrics, such as precision, recall, and F1-measure. Tables 11-13 show the binary classification of our proposed model in terms of precision, recall, and F1-measure, respectively. Tables 14-16 show the multi-attack classification evaluation in terms of precision, recall, and F1-measure. To further test the suitability of the proposed model for intrusion detection in MQTT-based smart IoT systems, we compared its performance with other classical machine learning models, such as DT, RF, NB, and KNN for binary and multi-class classification.

Tables 17 and 18 show the comparison of the proposed model with other classical machine learning models. The comparison was carried out using a number of parameters, such as accuracy, precision, recall, F1 measure, and training and testing times. The training and testing time in Tables 17 and 18 are measured in seconds. Depending on the specification of the system, the training and testing times may vary (we have already previously mentioned the system specifications). All of the classical machine learning algorithms mentioned in Tables 15 and 16 perform well on Uni-flow and Bi-flow data of MQTT protocol. However, on the Packet feature of MQTT data, our proposed model, in most cases, provides better results than other classical machine learning models. We also tested and compared the performance of our proposed model with other deep learning models', such as GRUs and LSTM, performance against MitM, Intrusion, and DoS over MQTT-enabled IoT network. One can see from Table 19 that the accuracy and F1-score of the proposed model are higher than other traditional learning models. 
Table 11. Binary-class attack classification results in terms of precision.

\begin{tabular}{|c|c|c|c|}
\hline Network Feature & Precision & Mean & Std \\
\hline Packet-flow & $\begin{array}{l}93.833 \\
93.752 \\
93.638 \\
93.943 \\
94.001\end{array}$ & 93.853 & 0.099 \\
\hline Bi-flow & $\begin{array}{l}99.911 \\
99.857 \\
99.873 \\
99.863 \\
99.902\end{array}$ & 99.881 & 0.021 \\
\hline Uni-flow & $\begin{array}{l}99.986 \\
99.914 \\
99.986 \\
99.972 \\
99.910\end{array}$ & 99.953 & 0.034 \\
\hline
\end{tabular}

Table 12. Binary-class attack classification results in terms of recall.

\begin{tabular}{cllc}
\hline Network Feature & Recall & Mean & Std \\
\hline & 86.733 & & \\
Packet-flow & 86.852 & & 0.119 \\
& 86.648 & 86.815 & \\
& 86.843 & & \\
& 87.001 & & \\
Bi-flow & 88.711 & & \\
& 88.697 & & \\
& 88.693 & & \\
& 88.720 & & \\
& 88.675 & & \\
& 87.996 & & \\
& 88.110 & & \\
\hline
\end{tabular}

Table 13. Binary-class attack classification results in term of F1-score.

\begin{tabular}{cccc}
\hline Network Feature & F1 Score & Mean & Std \\
\hline Packet-flow & 90.143 & & 0.118 \\
& 90.170 & 90.188 & \\
& 90.007 & & 0.011 \\
Bi-flow & 90.253 & & \\
& 90.365 & 93.958 & \\
& 93.978 & & \\
& 93.946 & & \\
Uni-flow & 93.951 & & 0.025 \\
\end{tabular}


Table 14. Multi-class attacks classification results in term of precision.

\begin{tabular}{cccc}
\hline Network Feature & Precision & Mean & Std \\
\hline & 89.693 & & \\
Packet-flow & 90.970 & 89.4118 & \\
& 88.187 & & \\
& 88.099 & & \\
& 90.110 & & \\
Bi-flow & 95.378 & 95.109 & \\
& 95.116 & & \\
& 94.991 & & \\
& 94.988 & & \\
& 95.074 & & \\
& 94.654 & & \\
\hline
\end{tabular}

Table 15. Multi-class attacks classification results in term of recall.

\begin{tabular}{cllc}
\hline Network Feature & Recall & Mean & Std \\
\hline & 81.385 & & \\
Packet-flow & 81.970 & & \\
& 82.107 & & \\
& 80.999 & & \\
& 81.743 & & \\
Bi-flow & 86.331 & & \\
& 87.116 & & \\
& 86.591 & & \\
& 86.222 & & \\
& 87.324 & & \\
\hline & 85.966 & & \\
\hline
\end{tabular}

Table 16. Multi-class attacks classification results in term of F1-score.

\begin{tabular}{cccc}
\hline Network Feature & F1 Score & Mean & Std \\
\hline & 85.337 & & \\
Packet-flow & 86.235 & 85.346 & 0.620 \\
& 85.038 & & \\
& 84.399 & & \\
& 85.722 & & 0.235 \\
Bi-flow & 90.629 & 90.718 & \\
& 90.940 & & \\
& 90.596 & & \\
& 90.393 & & \\
& 91.034 & & \\
Uni-Flow & 90.101 & & \\
& 90.560 & & \\
& 90.798 & & \\
& 90.364 & & \\
& 90.201 & & \\
\hline
\end{tabular}


Table 17. Performance comparison of the proposed DL-based IDS with other ML algorithm-based IDSs (binary-class classification).

\begin{tabular}{|c|c|c|c|c|c|c|c|}
\hline Dataset & Models & Accuracy & Precision & Recall & F1 Score & Training Time (s) & Testing Time (s) \\
\hline \multirow{5}{*}{ Packet-flow } & K-NN & 83.980 & 80.643 & 78.654 & 79.636 & 63.33 & 14.47 \\
\hline & NB & 49.780 & 45.503 & 50.003 & 47.646 & 7.70 & 4.41 \\
\hline & DT & 90.970 & 91.211 & 89.108 & 90.147 & 9.10 & 4.18 \\
\hline & $\mathrm{RF}$ & 89.985 & 90.345 & 88.976 & 89.655 & 10.37 & 4.65 \\
\hline & Proposed & 94.943 & 93.853 & 86.815 & 90.188 & 195.83 & 6.10 \\
\hline \multirow{5}{*}{ Bi-flow } & K-NN & 98.999 & 99.673 & 81.991 & 89.971 & 53.77 & 12.30 \\
\hline & NB & 94.188 & 94.007 & 80.693 & 86.843 & 5.80 & 3.20 \\
\hline & DT & 98.698 & 99.467 & 84.377 & 91.303 & 7.80 & 3.15 \\
\hline & RF & 99.537 & 99.631 & 84.117 & 91.219 & 8.10 & 3.35 \\
\hline & Proposed & 99.753 & 99.881 & 88.699 & 93.958 & 168.83 & 4.70 \\
\hline \multirow{5}{*}{ Uni-flow } & K-NN & 99.125 & 99.116 & 98.023 & 98.566 & 48.88 & 11.55 \\
\hline & NB & 90.065 & 88.213 & 70.003 & 78.060 & 6.92 & 3.90 \\
\hline & DT & 99.111 & 99.345 & 97.932 & 98.633 & 7.39 & 3.05 \\
\hline & $\mathrm{RF}$ & 99.061 & 99.111 & 97.999 & 98.551 & 8.51 & 3.83 \\
\hline & Proposed & 99.144 & 98.395 & 98.984 & 98.688 & 145.68 & 4.90 \\
\hline
\end{tabular}

Table 18. Performance comparison of proposed DL-based IDS with other ML algorithm-based IDSs (multi-class classification).

\begin{tabular}{|c|c|c|c|c|c|c|c|}
\hline Dataset & Models & Accuracy & Precision & Recall & F1 Score & Training Time (s) & Testing Time (s) \\
\hline \multirow{5}{*}{ Packet-flow } & K-NN & 81.333 & 78.993 & 77.991 & 78.489 & 61.74 & 13.90 \\
\hline & NB & 48.786 & 70.332 & 49.156 & 57.868 & 7.20 & 3.90 \\
\hline & DT & 90.539 & 89.832 & 79.195 & 84.178 & 8.90 & 4.37 \\
\hline & RF & 89.865 & 88.991 & 81.327 & 84.987 & 9.30 & 3.98 \\
\hline & Proposed & 90.798 & 89.4118 & 81.640 & 85.346 & 198.45 & 6.35 \\
\hline \multirow{5}{*}{ Bi-flow } & K-NN & 96.976 & 88.321 & 82.333 & 85.221 & 54.80 & 11.90 \\
\hline & NB & 91.188 & 91.996 & 79.375 & 85.220 & 6.64 & 3.82 \\
\hline & DT & 97.039 & 95.479 & 80.998 & 87.644 & 6.10 & 2.89 \\
\hline & RF & 97.979 & 96.391 & 84.373 & 89.982 & 8.39 & 3.97 \\
\hline & Proposed & 98.127 & 95.109 & 86.717 & 90.718 & 163.27 & 4.44 \\
\hline \multirow{5}{*}{ Uni-flow } & K-NN & 96.992 & 96.973 & 76.111 & 85.284 & 46.88 & 10.98 \\
\hline & NB & 70.765 & 69.185 & 70.988 & 70.075 & 6.49 & 3.46 \\
\hline & DT & 96.171 & 96.140 & 75.899 & 84.828 & 7.70 & 2.98 \\
\hline & $\mathrm{RF}$ & 95.297 & 95.117 & 73.995 & 83.236 & 8.29 & 3.37 \\
\hline & Proposed & 97.084 & 94.761 & 86.435 & 90.605 & 143.38 & 4.58 \\
\hline
\end{tabular}

Table 19. Comparison with other deep learning models.

\begin{tabular}{ccc}
\hline Models & Accuracy & F1 Score \\
\hline LSTM [24] & 0.9337 & 0.9328 \\
GRUs [24] & 0.9608 & 0.9577 \\
Proposed & 0.9713 & 0.9599 \\
\hline
\end{tabular}


(A) Binary Classification results

LosS

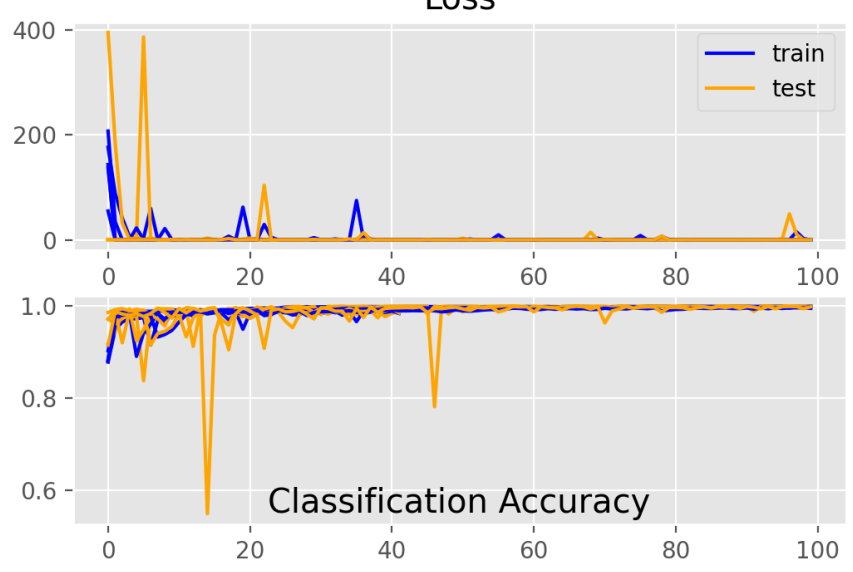

(a) Uni-flow: mean $=99.927 \mathrm{std}=0.030, \mathrm{n}=5$

\section{Loss}

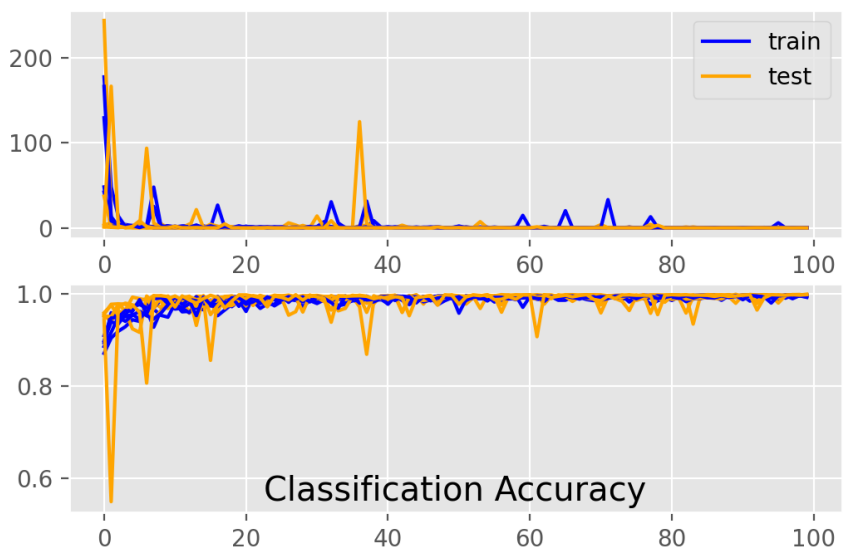

(b) Bi-flow: mean $=99.753$ std $=0.029, \mathrm{n}=5$

Loss
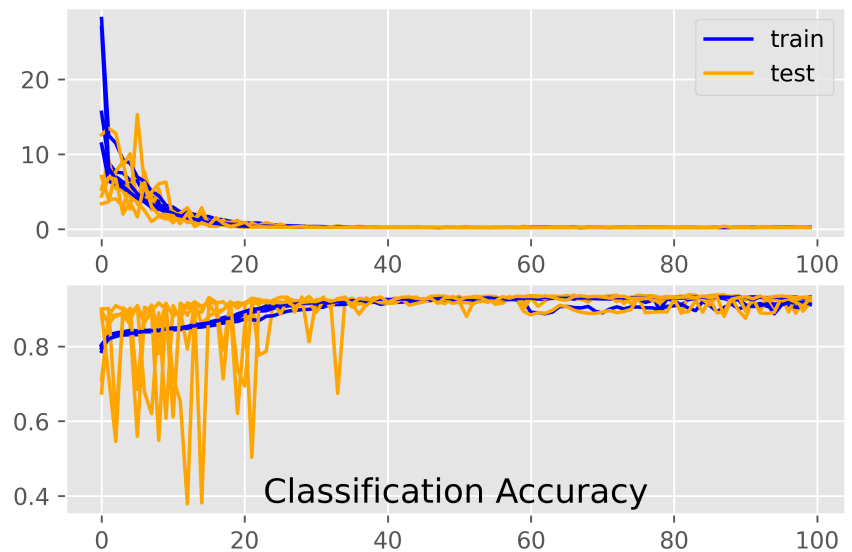

(c) Packet-flow: mean $=94.943$ std $=0.034, \mathrm{n}=5$
(B) Binary Classification results

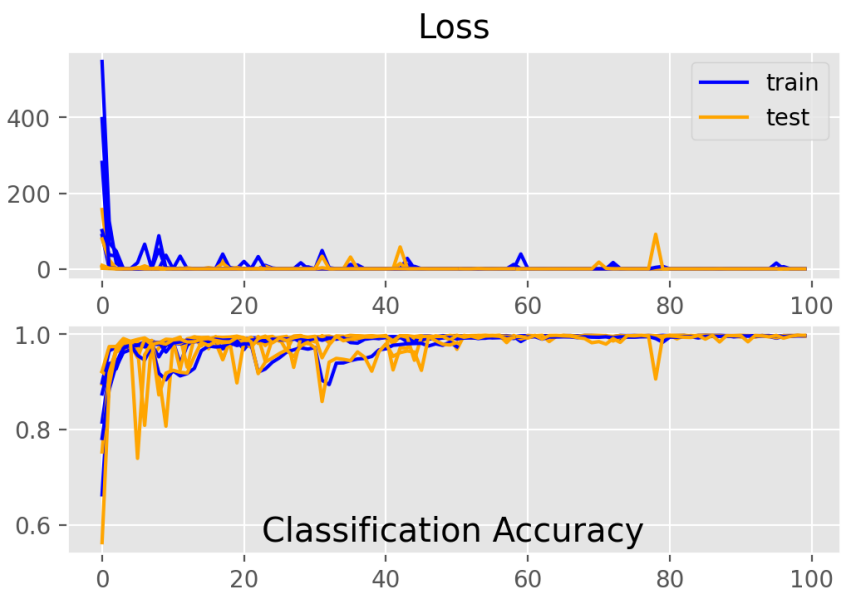

(d) Uni-flow: mean $=97.084 \mathrm{std}=0.524, \mathrm{n}=5$

Loss
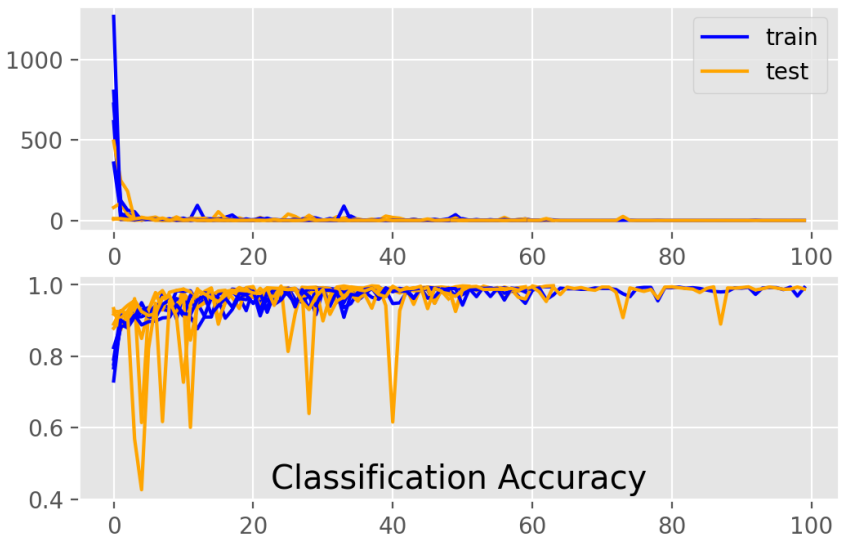

(e) Bi-flow:mean $=98.127 \mathrm{std}=0.154, \mathrm{n}=5$

Loss

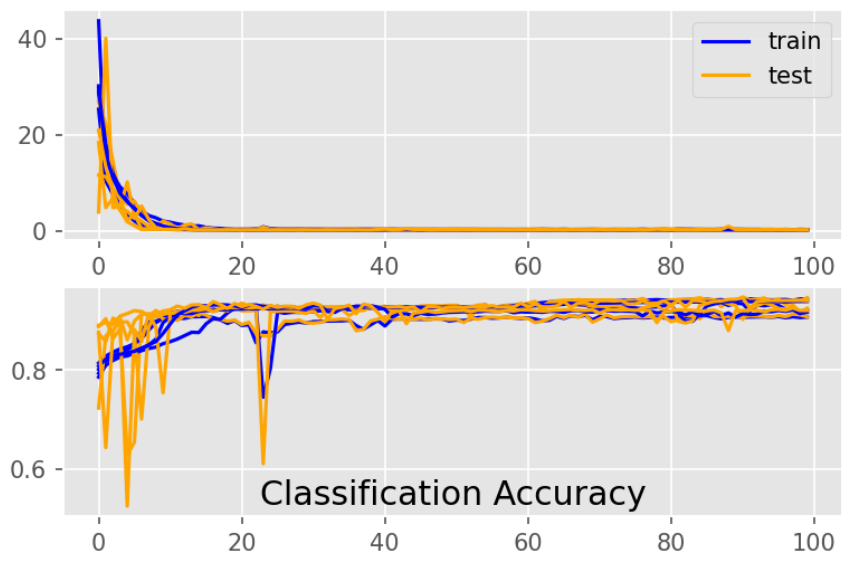

(f) Packet-flow: mean $=90.798$ std $=2.498, \mathrm{n}=5$

Figure 9. Five-fold cross validated accuracies for 3 abstraction-level features of MQTT.

\section{Conclusions and Future Direction}

This paper presents a DNN-based intrusion detection system for MQTT-enabled IoT smart systems. A recently published MQTT-IoT-IDS2020 and another MQTT dataset are used to evaluate the performance of the proposed model. The MQTT-IoT-IDS2020 dataset contains three abstraction-level features of MQTT-enabled IoT, including Packet- 
flow, Bi-flow, and Uni-flow features. There are five files in each of these featured data representing attack and normal scenarios. The data were organised such that each separated feature gets a subset in order to assess the performance in binary-class and multi-class attack classification. The tests were conducted under different batch sizes, such as 32,64 , and 128, for binary and multi-classifications. The results show that increasing the batch size of the training subset improves the convergence and performance of the classifier. The performance of the proposed DL-based IDS with a default learning rate and using the ADAM optimiser was compared with the performance of conventional ML-based IDSs, including KNN, NB, DT, and RF. Furthermore, the proposed model was tested for binary-class as well as multi-class attack classification with different activation functions at the output layers. The results show that the DL-based model for Bi-flow and Uni-flow featured data can achieve 99\% accuracy and 98\% accuracy for binary and multi-class attack classification, respectively. However, in Packet-flow featured data, the accuracy for binary and multi-class were $94 \%$ and $90 \%$, respectively. Additionally, we also tested the performance of the proposed model against DoS and MitM, etc., over an MQTT-based IoT system. From the results and comparison tables, it was evident that the proposed model has higher accuracy than other state-of-the-art deep learning models. In the future, we intend to investigate the vulnerability of new types of attacks on various IoT protocols. Our aim is to propose a novel deep learning-based model for new vulnerabilities.

Author Contributions: Conceptualisation, M.A.K. (Muhammad Almas Khan), M.A.K. (Muazzam A. Khan), S.S.J., A.A.S. and J.A.; Experimental analysis, M.A.K. (Muhammad Almas Khan); Writingoriginal draft preparation, M.A.K. (Muhammad Almas Khan), S.U.J. and S.S.J.; Review and editing, M.A.K. (Muazzam A. Khan) and J.A.; Supervision, M.A.K. (Muazzam A. Khan), S.U.J., J.A., N.P. and W.J.B. All authors have read and agreed to the published version of the manuscript.

Funding: This research received no external funding.

Institutional Review Board Statement: Not Applicable.

Informed Consent Statement: Not Applicable.

Data Availability Statement: The publicly available data set can be found at: https:/ /joseaveleira.es / dataset and https: / / ieee-dataport.org/open-access / mqtt-iot-ids2020-mqtt-internet-things-intrusiondetection-dataset.

Acknowledgments: One of the authors, Sajjad Shaukat Jamal, extends his gratitude to the Deanship of Scientific Research at King Khalid University for funding this work through a research groups program under grant number R. G. P. 1/77/42.

Conflicts of Interest: The authors declare no conflict of interest.

\section{References}

1. Al-Masri, E.; Kalyanam, K.R.; Batts, J.; Kim, J.; Singh, S.; Vo, T.; Yan, C. Investigating messaging protocols for the Internet of Things (IoT). IEEE Access 2020, 8, 94880-94911. [CrossRef]

2. Kodali, R.K.; Soratkal, S. MQTT based home automation system using ESP8266. In Proceedings of the 2016 IEEE Region 10 Humanitarian Technology Conference (R10-HTC), Agra, India, 21-23 December 2016; pp. 1-5.

3. Cornel-Cristian, A.; Gabriel, T.; Arhip-Calin, M.; Zamfirescu, A. Smart home automation with MQTT. In Proceedings of the 2019 54th International Universities Power Engineering Conference (UPEC), Bucharest, Romania, 3-6 September 2019 ; pp. 1-5.

4. Prabaharan, J.; Swamy, A.; Sharma, A.; Bharath, K.N.; Mundra, P.R.; Mohammed, K.J. Wireless home automation and security system using MQTT protocol. In Proceedings of the 2017 2nd IEEE International Conference on Recent Trends in Electronics, Information \& Communication Technology (RTEICT), Bangalore, India, 19-20 May 2017; pp. 2043-2045.

5. Kodali, R.K.; Sarjerao, B.S. A low cost smart irrigation system using MQTT protocol. In Proceedings of the 2017 IEEE Region 10 Symposium (TENSYMP), Cochin, India, 14-16 July 2017; pp. 1-5.

6. Mukherji, S.V.; Sinha, R.; Basak, S.; Kar, S.P. Smart agriculture using internet of things and mqtt protocol. In Proceedings of the 2019 International Conference on Machine Learning, Big Data, Cloud and Parallel Computing (COMITCon), Faridabad, India, 14-16 February 2019; pp. 14-16.

7. Atmoko, R.A.; Yang, D. Online monitoring \& controlling industrial arm robot using mqtt protocol. In Proceedings of the 2018 IEEE International Conference on Robotics, Biomimetics, and Intelligent Computational Systems (Robionetics), Bandung, Indonesia, 8-10 August 2018; pp. 12-16. 
8. Safaei, B.; Monazzah, A.M.H.; Bafroei, M.B.; Ejlali, A. Reliability side-effects in Internet of Things application layer protocols. In Proceedings of the 2017 2nd International Conference on System Reliability and Safety (ICSRS), Milan, Italy, 20-22 December 2017; pp. 207-212.

9. Soni, D.; Makwana, A. A survey on mqtt: A protocol of internet of things (iot). In Proceedings of the International Conference on Telecommunication, Power Analysis And Computing Techniques (ICTPACT-2017), Chennai, India, 6-8 April 2017 ; Volume 20.

10. Hunkeler, U.; Truong, H.L.; Stanford-Clark, A. MQTT-S-A publish/subscribe protocol for Wireless Sensor Networks. In Proceedings of the 2008 3rd International Conference on Communication Systems Software and Middleware and Workshops (COMSWARE'08), Bangalore, India, 6-10 January 2008; pp. 791-798.

11. Zriqat, A.I.; Altamimi, A.M. Security and privacy issues in eHealthcare systems: Towards trusted services. Int. J. Adv. Comput. Sci. Appl. 2016, 7, 229-236.

12. Mathur, A.; Newe, T.; Rao, M. Defence against black hole and selective forwarding attacks for medical WSNs in the IoT. Sensors 2016, 16, 118. [CrossRef] [PubMed]

13. Butt, S.A.; Diaz-Martinez, J.L.; Jamal, T.; Ali, A.; De-La-Hoz-Franco, E.; Shoaib, M. IoT smart health security threats. In Proceedings of the 2019 19th International Conference on Computational Science and Its Applications (ICCSA), St. Petersburg, Russia, 1-4 July 2019; pp. 26-31.

14. Markowsky, L.; Markowsky, G. Scanning for vulnerable devices in the Internet of Things. In Proceedings of the 2015 IEEE 8th International Conference on Intelligent Data Acquisition and Advanced Computing Systems: Technology and Applications (IDAACS), Warsaw, Poland, 24-26 September 2015; Volume 1, pp. 463-467.

15. Andy, S.; Rahardjo, B.; Hanindhito, B. Attack scenarios and security analysis of MQTT communication protocol in IoT system. In Proceedings of the 2017 4th International Conference on Electrical Engineering, Computer Science and Informatics (EECSI), Yogyakarta, Indonesia, 19-21 September 2017; pp. 1-6.

16. Gujar, N.S. Message Queuing Telemetry Transport (MQTT) based hybrid smart streetlight system for smart applications. In Proceedings of the 2019 International Conference on Smart Systems and Inventive Technology (ICSSIT), Tirunelveli, India, 27-29 November 2019; pp. 832-836.

17. Vaccari, I.; Aiello, M.; Cambiaso, E. SlowITe, a novel denial of service attack affecting MQTT. Sensors 2020, 20, 2932. [CrossRef]

18. Firdous, S.N.; Baig, Z.; Valli, C.; Ibrahim, A. Modelling and evaluation of malicious attacks against the iot mqtt protocol. In Proceedings of the 2017 IEEE International Conference on Internet of Things (iThings) and IEEE Green Computing and Communications (GreenCom) and IEEE Cyber, Physical and Social Computing (CPSCom) and IEEE Smart Data (SmartData), Exeter, UK, 21-23 June 2017; pp. 748-755.

19. Potrino, G.; De Rango, F.; Santamaria, A.F. Modeling and evaluation of a new IoT security system for mitigating DoS attacks to the MQTT broker. In Proceedings of the 2019 IEEE Wireless Communications and Networking Conference (WCNC), Marrakesh, Morocco, 15-18 April 2019; pp. 1-6.

20. Al Enany, M.O.; Harb, H.M.; Attiya, G. A Comparative analysis of MQTT and IoT application protocols. In Proceedings of the 2021 International Conference on Electronic Engineering (ICEEM), Menouf, Egypt, 3-4 July 2021; pp. 1-6.

21. Mahdavinejad, M.S.; Rezvan, M.; Barekatain, M.; Adibi, P.; Barnaghi, P.; Sheth, A.P. Machine learning for Internet of Things data analysis: A survey. Digit. Commun. Netw. 2018, 4, 161-175. [CrossRef]

22. da Costa, K.A.; Papa, J.P.; Lisboa, C.O.; Munoz, R.; de Albuquerque, V.H.C. Internet of Things: A survey on machine learningbased intrusion detection approaches. Comput. Netw. 2019, 151, 147-157. [CrossRef]

23. Chaabouni, N.; Mosbah, M.; Zemmari, A.; Sauvignac, C.; Faruki, P. Network intrusion detection for IoT security based on learning techniques. IEEE Commun. Surv. Tutor. 2019, 21, 2671-2701. [CrossRef]

24. Alaiz-Moreton, H.; Aveleira-Mata, J.; Ondicol-Garcia, J.; Muñoz-Castañeda, A.L.; García, I.; Benavides, C. Multiclass classification procedure for detecting attacks on MQTT-IoT protocol. Complexity 2019, 2019, 6516253 . [CrossRef]

25. Hindy, H.; Bayne, E.; Bures, M.; Atkinson, R.; Tachtatzis, C.; Bellekens, X. Machine learning based IoT Intrusion Detection System: An MQTT case study (MQTT-IoT-IDS2020 Dataset). In Proceedings of the International Networking Conference, Online, 19-21 September 2020; Springer: Cham, Switzerland, 2020; pp. 73-84.

26. Ahmadon, M.A.B.; Yamaguchi, N.; Yamaguchi, S. Process-Based Intrusion Detection Method for IoT System with MQTT Protocol. In Proceedings of the 2019 IEEE 8th Global Conference on Consumer Electronics (GCCE), Osaka, Japan, 15-18 October 2019; pp. 953-956.

27. Haripriya, A.; Kulothungan, K. Secure-MQTT: An efficient fuzzy logic-based approach to detect DoS attack in MQTT protocol for internet of things. EURASIP J. Wirel. Commun. Netw. 2019, 2019, 90.

28. Jan, S.U.; Lee, Y.D.; Koo, I.S. A distributed sensor-fault detection and diagnosis framework using machine learning. Inf. Sci. 2021, 547, 777-796. [CrossRef]

29. Mohammadi, M.; Rashid, T.A.; Karim, S.H.T.; Aldalwie, A.H.M.; Tho, Q.T.; Bidaki, M.; Rahmani, A.M.; Hoseinzadeh, M. A comprehensive survey and taxonomy of the SVM-based intrusion detection systems. J. Netw. Comput. Appl. 2021, 178, 102983. [CrossRef]

30. Jan, S.U.; Vu, V.H.; Koo, I.S. Performance analysis of support vector machine-based classifier for spectrum sensing in cognitive radio networks. In Proceedings of the 2018 International Conference on Cyber-Enabled Distributed Computing and Knowledge Discovery (CyberC), Zhengzhou, China, 18-20 October 2018; pp. 385-3854. 
31. Abdel-Basset, M.; Hawash, H.; Chakrabortty, R.K.; Ryan, M.J. Semi-supervised Spatio-Temporal Deep Learning for Intrusions Detection in IoT Networks. IEEE Internet Things J. 2021, 8, 12251-12265. [CrossRef]

32. Faker, O.; Dogdu, E. Intrusion detection using big data and deep learning techniques. In Proceedings of the 2019 ACM Southeast Conference, Kennesaw, GA, USA, 18-20 April 2019; pp. 86-93.

33. Ciklabakkal, E.; Donmez, A.; Erdemir, M.; Suren, E.; Yilmaz, M.K.; Angin, P. ARTEMIS: An intrusion detection system for MQTT attacks in Internet of Things. In Proceedings of the 2019 38th Symposium on Reliable Distributed Systems (SRDS), Lyon, France, 1-4 October 2019; pp. 369-3692.

34. Vaccari, I.; Chiola, G.; Aiello, M.; Mongelli, M.; Cambiaso, E. MQTTset, a New Dataset for Machine Learning Techniques on MQTT. Sensors 2020, 20, 6578. [CrossRef]

35. Vinayakumar, R.; Alazab, M.; Soman, K.; Poornachandran, P.; Al-Nemrat, A.; Venkatraman, S. Deep learning approach for intelligent intrusion detection system. IEEE Access 2019, 7, 41525-41550. [CrossRef]

36. Zhong, M.; Zhou, Y.; Chen, G. Sequential model based intrusion detection system for IoT servers using deep learning methods. Sensors 2021, 21, 1113. [CrossRef]

37. Jothi, B.; Pushpalatha, M. WILS-TRS-A novel optimized deep learning based intrusion detection framework for IoT networks. Pers. Ubiquitous Comput. 2021. [CrossRef]

38. Maseer, Z.K.; Yusof, R.; Bahaman, N.; Mostafa, S.A.; Foozy, C.F.M. Benchmarking of Machine Learning for Anomaly Based Intrusion Detection Systems in the CICIDS2017 Dataset. IEEE Access 2021, 9, 22351-22370. [CrossRef]

39. Ullah, I.; Ullah, A.; Sajjad, M. Towards a Hybrid Deep Learning Model for Anomalous Activities Detection in Internet of Things Networks. IoT 2021, 2, 428-448. [CrossRef]

40. Shalaginov, A.; Semeniuta, O.; Alazab, M. MEML: Resource-aware MQTT-based machine learning for network attacks detection on IoT edge devices. In Proceedings of the 12th IEEE/ACM International Conference on Utility and Cloud Computing Companion, Auckland, New Zealand, 2-5 December 2019; pp. 123-128.

41. Berner, J.; Grohs, P.; Kutyniok, G.; Petersen, P. The Modern Mathematics of Deep Learning. arXiv 2021, arXiv:2105.04026.

42. Ghasemi, J.; Esmaily, J.; Moradinezhad, R. Intrusion detection system using an optimized kernel extreme learning machine and efficient features. Sādhanā 2020, 45, 1-9. [CrossRef]

43. Liu, Z.; Ghulam, M.U.D.; Zhu, Y.; Yan, X.; Wang, L.; Jiang, Z.; Luo, J. Deep learning approach for IDS. In Fourth International Congress on Information and Communication Technology; Springer: Singapore, 2020; pp. 471-479.

44. Sarnovsky, M.; Paralic, J. Hierarchical intrusion detection using machine learning and knowledge model. Symmetry 2020, $12,203$. [CrossRef]

45. de Souza, C.A.; Westphall, C.B.; Machado, R.B.; Sobral, J.B.M.; dos Santos Vieira, G. Hybrid approach to intrusion detection in fog-based IoT environments. Comput. Netw. 2020, 180, 107417. [CrossRef]

46. Ibitoye, O.; Shafiq, O.; Matrawy, A. Analyzing Adversarial Attacks against Deep Learning for Intrusion Detection in IoT Networks. In Proceedings of the 2019 IEEE Global Communications Conference (GLOBECOM), Waikoloa, HI, USA, 9-13 December 2019; pp. 1-6. [CrossRef]

47. Alsaedi, A.; Moustafa, N.; Tari, Z.; Mahmood, A.; Anwar, A. TON_IoT telemetry dataset: A new generation dataset of IoT and IIoT for data-driven Intrusion Detection Systems. IEEE Access 2020, 8, 165130-165150. [CrossRef]

48. Dataset. Available online: https://joseaveleira.es/dataset (accessed on 8 July 2021).

49. DoS 2017 | Datasets | Research I Canadian Institute for Cybersecurity / UNB. Available online: https://www.unb.ca/cic/ datasets / dos-dataset.html (accessed on 8 July 2021).

50. Sharafaldin, I.; Lashkari, A.H.; Ghorbani, A.A. Toward generating a new intrusion detection dataset and intrusion traffic characterization. ICISSp 2018, 1, 108-116.

51. Zikria, Y.B.; Yu, H.; Afzal, M.K.; Rehmani, M.H.; Hahm, O. Internet of things (IoT): Operating system, applications and protocols design, and validation techniques. Future Gener. Comput. Syst. 2018, 88, 699-706. [CrossRef]

52. Alouache, L.; Nguyen, N.; Aliouat, M.; Chelouah, R. Survey on IoV routing protocols: Security and network architecture. Int. J. Commun. Syst. 2019, 32, e3849. [CrossRef]

53. Igure, V.M.; Laughter, S.A.; Williams, R.D. Security issues in SCADA networks. Comput. Secur. 2006, 25, 498-506. [CrossRef]

54. Misbahuddin, S. Fault tolerant remote terminal units (RTUs) in SCADA systems. In Proceedings of the 2010 International Symposium on Collaborative Technologies and Systems, Chicago, IL, USA, 17-21 May 2010; pp. 440-446.

55. Kingma, D.P.; Ba, J. Adam: A method for stochastic optimization. arXiv 2014, arXiv:1412.6980.

56. Duchi, J.; Hazan, E.; Singer, Y. Adaptive subgradient methods for online learning and stochastic optimization. J. Mach. Learn. Res. 2011, 12, 2121-2159.

57. Ruder, S. An overview of gradient descent optimization algorithms. arXiv 2016, arXiv:1609.04747.

58. Kandel, I.; Castelli, M. The effect of batch size on the generalizability of the convolutional neural networks on a histopathology dataset. ICT Express 2020, 6, 312-315. [CrossRef]

59. Hoffer, E.; Hubara, I.; Soudry, D. Train longer, generalize better: Closing the generalization gap in large batch training of neural networks. arXiv 2017, arXiv:1705.08741.

60. Smith, S.L.; Kindermans, P.J.; Ying, C.; Le, Q.V. Don't decay the learning rate, increase the batch size. arXiv 2017, arXiv:1711.00489. 